$$
\text { MASTER }
$$

\title{
Geostatistics Project of the \\ National Uranium Resource Evaluation Program
}

October 1979-March 1980

\author{
K. Campbell \\ T. R. Bement \\ J. A. Howell \\ R. J. Beckman \\ K. Jackson \\ P. Buslee
}




\author{
GEOSTATI!. ':CS PROJECT OF THE \\ NATIONAL URANIUM RESOURCE EVALUATION PROGRAM \\ OCTOBER 1979 - MARCH 1980 \\ by \\ K. Campbell, T. R. Bement, J. A. Howell, R. J. Beckman, \\ $K$. Jackson and P. Buslee
}

\begin{abstract}
During the period covered by this report, the authors investigated the serial properties of aerial radiometric data. Results were applied to the choice of minimum segment width in the maximum variance segments algorithm and to the use of aerial radiometric data in the design of ground sampling experiments.

The report also presents the results of a comparison of normal and lognormal percentile estimation techniques.

Twenty-two quadrangles are being analyzed in the search for a uranium favorability index. Computer codes developed during this investigation have been provided to the Bendix Field Engineering Corporation in Grand Junction, Colorado.
\end{abstract}

\title{
I. INTRODUCTION
}

This report outlines the activities and progress of the Los Alamos Scientific Laboratory (LASL) on the Geostatistics project during the first half of FY1980. The Geostatistics project is part of the National Uranium Resource Evaluation (NURE) program sponsored by the US Department of Energy (DOE), Grand Junction, Colorado, office. The NURE program is designed to assess the potential uranium resources throughout the conterminous United States and Alaska. In close cooperation with the Grand Junction Office of DOE, the Geostatistics project at LASL applies statistical methods to the analysis of data collected by airborne instrumentation. To handle a broad range of problems 
related to the NURE, LASL maintains a close statistical consulting relationship with the DOE Grand Junction Office and the Bendix Field Engineering Corporation (BFEC) in Grand Junction.

We developed a statistical model for the correlation between observations taken along a flight line and considered two applications. The first inolves the choice of a minimum segment width for the maximum variance segments algorithm being tested by BFEC. The second application irvolves the use of aerial radiometric data to aid in the design of ground-based sampling experiments. This last application developed as a result of a joint BFEC-LASL effort to design a statistically valid ground-based experiment in East Texas.

Standard deviation maps that are produced as part of the aerial program are closely related to the estimation of percentiles in the normal and lognormal probability distributions. This, plus the fact that anomalies are often defined explicitly or implicitly in terms of percentiles, motivated a study to determine the effect of using the wrong distribution (normal instead of lognormal or vice versa) when estimating percentiles.

Twenty-two quadrangles with assigned uranium favorabilities are being analyzed in the search for a uranium favorability index. Computer codes developed during this investigation have been transferred to BFEC. 


\section{NOTES ON THE TWO "MAXIMUM VARIANCE SEGMENTS" ALGORITHMS}

In Ref. 1 Bement and Waterman give (1) an algorithm for determining the largest sum $S_{k}(N)$ of the variances of up to $k$ disjoint segments from the data $x_{1}, x_{2}, \ldots, x_{N}$, and (2) a much simpler algorithm for determining $S(N)=\sup S_{k}(N)$.

The second algorithm is incorrectly stated; it should read

$$
\begin{aligned}
& S(0)=0 \\
& S(j)=\max _{1 \leq \ell \leq j}\{S(\ell-1)+v(\ell, j)\},
\end{aligned}
$$

where $v(j, j)=0$ by definition. When modified to allow only segments of length between $w$ and $w$, this algorithm becomes

$$
\begin{aligned}
& S(j)=0,0 \leq j<w \\
& S(j)=\max \left\{S\left(j-\frac{1}{\ell}\right), \max _{1}[j) \leq \ell<\ell_{2}(j),\right.
\end{aligned}
$$

where $\ell_{1}(j)=\max (1, j-w+1)$ and $\ell_{2}(j)=j-w+1$.

Given $S(j), 1 \leq j \leq N$, plus, for each $j$, a flag $L(j)$ defined by

$$
L(j)=\left\{\begin{array}{l}
0 \text { if } S(j)=S(j-1) \\
\ell \text { if } S(j)=S(\ell-1)+v(\ell, j),
\end{array}\right.
$$

it is possible to find the segments which went into $S(N)$, the maximum possible total sum of variances for segments of the data. This is the algorithm implemented by a program that BFEC is usina.

Similarly, given $S_{k}(j), 1 \leq j \leq N$, as computed by the first algorithm, and a flag $L_{k}(j)$ defined by

$$
L_{k}(j)=\left\{\begin{array}{l}
0 \text { if } S_{k}(j)=S_{k-1}(j) \\
\ell \text { if } S_{k}(j)=S_{k-1}(\ell-1)+v(\ell, j),
\end{array}\right.
$$


one can work backwards to find the best $k$ segments. This algorithm is much harder to implement, however, and requires either much more computer time or a large random-access memory such as "extended core" on the Control Data Corporation machines.

These two algorithms select segmerts of data on different principles, and there is no reason to suppose that the $k$ segments with largest variance produced by the second algorithm will be the $k$ segments that go into $S_{k}(N)$ as computed by the first algorithm. In fact, unless $k$ is very large, on the order of $N / w$, this will hardly ever be the case.

The first algorithm picks segments to maximize the total variance achieved by exactly $k$ segments. The second algorithm, on the other hand, maximizes the total variance achievable by any number of segments. For noisy data, the variance is relatively independent of the length of the segment, and therefore the way to maximize

$$
\sum_{j=1}^{k} v[\ell(j), j]
$$

is to pick as many segments as possible in $x_{1}, \ldots, x_{N}$, which means picking them as short as possible. For such data, therefore, the second algorithm almost invariably selects segments of the minimum length, $w$, and never gets beyond $w+2$ or $w+3$.

Interestingly enough, although the first algorithm has no such built-in bias toward short segments provided $k \ll \mathrm{N} / \mathrm{w}$, it also appears to choose segments of the minimum length more frequently than expected, given that the expected variance of the segment increases with the length of the segment. (This is for correlated data and an assumption that we have something besides noise, although the noise is large). However, it chooses somewhat different segments (although it identifies, in a general way, the same regions pulled out by the second algorithm). As the minimum segment width, $w$, increases, the first algorithm appears to have a greater tendency to choose segments of length greater than $w$, and the sum of the variances of the top $k$ segments found by the second algorithin becomes a smaller fraction of $S_{k}(N)$.

Table I shows the ton ten seoments in a string of $n=560$ observations from one geological type selected by the first algorithm, where the length of the segment was restricted to lie between 5 and 15 seconds of data. Table II shows the results from the second algorithm, similarly restricted. Roughly 
similar areas are identified, but the chosen segments are not identical, and the second algorithm has clearly made a suboptimal choice, with total variance of 1103.1 in ten segments, compared with $S_{10}(\mathrm{~N})=1177.0$ computed by the first algorithm. In particular, the second algorithm appears to have missed a fairly significant segment, 467-471, chosen sixth by the first algorithm. It also selected two segments, 253-257 and 260-264, near the first algorithm's fourth choice, 256-260.

Tables III and IV represent a similar comparison for segments between 10 and 25 seconds in length. Here it is interesting to note that the first algorithm occasionally selects a very long segment, something which is almost impossible for the second algorithm. The second algorithm achieves a total variance of 790.4 in ten seaments, comoared with $S_{10}(N)=887.2$.

When applied to the NURE data, these discrepancies are probably not important, as long as the two algorithms identify approximately the same areas as interesting. There is some advantage to having all of the segments of about the same length, because then their variances are directly comparable, and we can justify picking off the top $k$, without worrying about the fact that the expected value of the variance increases with length.

TABLE I

SEGMENTS SELECTED BY FIRST ALGORI THM

$$
(w=5, w=15)
$$

\section{Endpoints}

548 - 553

$69-73$

19 - 23

256 - 260

$554-560$

467 - 471

$232-236$

$168-172$

$119-123$

$1-5$
Variance

199.1

125.9

122.4

121.6

111.8

108.5

100.5

96.9

96.7

93.5
Length

6

5

5

5

7

5

5

5

5

5

Note: Total variance $=S_{10}(N)=1177.0$. 
TABLE II

SEGMENTS SELECTED BY SECOND ALGORITHM

Endpoints

$$
\begin{array}{r}
549-553 \\
72-76 \\
554-560 \\
253-257 \\
231-235 \\
260-264 \\
168-172 \\
119-123 \\
16-20 \\
1-5
\end{array}
$$

Variance

188.1

119.3

111.8

105.8

100.2

97.0

96.9

96.7

93.7

93.5
Length

5
5
7
5
5
5
5
5
5
5

Note: Total variance $=1103.1$

TABLE III

SEGMENTS SELECTED BY FIRST ALGORITHM

$$
(w=10, w=25)
$$

Endpoints

$$
\begin{array}{r}
551-560 \\
69-78 \\
253-262 \\
39-53 \\
162-173 \\
404-428 \\
19-36 \\
117-126 \\
224-235 \\
1-10
\end{array}
$$

Variance

Length

$$
\begin{array}{r}
159.4 \\
97.5 \\
86.4 \\
84.5 \\
81.9 \\
78.8 \\
77.8 \\
75.8 \\
73.9 \\
71.4
\end{array}
$$

10

10

10

15

12

25

18

10

12

10

Note: Total variance $S_{10}(N)=887.2$. 
TABLE IV

SEGMENTS SELECTED BY SECOND ALGORITHM

$$
(w=10, w=25)
$$

Endpoints

$55 i-560$

256 - 265

$162-173$

$1-10$

$11-20$

$115-124$

$71-80$

$278-288$

$409-418$

$41-50$
Variance

159.4

836.4

81.9

71.4

70.8

67.9

66.9

65.6

61.8

58.4
Length

10

10

12

10

10

10

10

1 !.

10

10

Note: Total variance $=790.4$.

III. SERIAL PROPERTIES OF T:HE RADIOMETRIC DATA

In this section we describe a statistical model for the correlation between observations taken along a flight line. Physically the model is over-simplified, failing to take into account much of what is known about the processes of decay, gamma emission, scattering and measurement. However, a basic statistical model of this type will help us to see how the aerial data is related to what is on the ground, and in particular, what kinds of information might be extracted from the data. We consider applications related to the choice of a minimum "bin" or segment width for the maximum variance segments algorichm and the prior estimation of the components of variance in a ground-based survey.

\section{A. The Statistical Model}

Suppose that the gamma emissions lat a given frequency or in. a band of frequencies) at a point $(x, y)$ on the ground at time $t$ have intensity $x(x, y, t)$ counits per second per unit area. Let $E x(x, y, t)=\lambda(x, y)$, independent of time, where $\lambda(x, y)$ is presumably proportional to the concentration of the source element or elements at $(x, y)$. In turn, $\lambda(x, y)$ is assumed to be a realization of a spatial random process $\Lambda(x, y)$, and the principal task of the following 
development is to see how the properties of the $\Lambda$ process (e.g., its covariance function) are reflected in the aerial data.

(The notion that the concentration $\lambda(x, y)$ at a given point is a "realization" of a stochastic process is a statistical fiction which is very usefui in practice. For example, it underlies the work of Matheron, et al. (Ref. 2) and is similar to models used in other branches of statistics, for example, in sampling theory. There is nothing in the discussion which follows, however, that makes its use imperative, and the reader may prefer to think of the quantities which we denote using $E$ (expected values) as simply the appropriate spatial averages.)

The integrals of $x$ over a finite regiun and time interval,

$$
\int_{t}^{t+\Delta t} \iint_{S} x(x, y, \tau) d x d y d \tau,
$$

have Poisson distributions with mean

$$
\Delta t \iint_{S} \lambda(x, y) d x d y .
$$

Letting $S$ shrink to zero and $\Delta t \rightarrow 0$ implies that

$$
\begin{aligned}
& E[x(x, y, t) \mid \Lambda=\lambda]=\lambda(x, y) \\
& \operatorname{Var}[x(x, y, t) \mid \Lambda=\lambda]=\lambda(x, y),
\end{aligned}
$$

although $X$ itself is not Poisson (it does not have units of counts).

The number of photons reaching the airborne detector during the time interval $(t-\Delta t$ ) (which are recorded at time $t$ ) is a random quantity

$$
\begin{aligned}
K(t) & =\iint_{-\infty}^{\infty} \int_{t-\Delta t}^{t} p(x, y) x(v s-x,-y, s) d s d x d y+B(t) \\
& =I(t)+B(t),
\end{aligned}
$$


where $p(x, y)$ is the instrumental point spread function, usually modeled as the product of a frequency-dependent exponential term decreasing with altitude and a geometrical factor. The detector is assumed to be traveling with velocity, $v$, along the $x$-axis $(y=0) . B(t)$ is radiation from cosmic sources, from the aircraft, and so forth.

of the $k(t)$ photons, only

$$
z(t)=\varepsilon K(t)+\eta(t)
$$

are actually recorded, where $\varepsilon$ is the efficiency of the detector and $n$ is a random component. This recording process might be modeled as a binomial, $Z(t) \sim B[K(t), \varepsilon]$, or a Poisson process, $Z(t) \sim P[\varepsilon K(t)]$.

We begin by assuming that $\Lambda(x, y)$ is a second-order stationary process, which means that

$$
\begin{aligned}
& E \Lambda(x, y)=m=\text { constant for all }(\Lambda, y) \text {, and } \\
& \begin{aligned}
\operatorname{Cov}[\Lambda(x, y), \Lambda(x+\Delta x, y+\Delta y)]= & \left.E_{\{}[\Lambda(x, y)-m][\Lambda(x+\Delta x, y+\Delta y)-m]\right\} \\
= & C_{\Lambda}(\Delta x, \Delta y),
\end{aligned}
\end{aligned}
$$

a function which depends only on the separation $(\Delta x, \Delta y)$ of two points, not on the absolute position $(x, y)$. If the background $B(t)$ is also a stationary process, then so is $z(t)$. In particuiar,

$$
\begin{aligned}
E Z(t) & =E[I(t)+B(t)] \\
& =m P_{v}+E B(t),
\end{aligned}
$$

where

$$
P_{v}=\iint_{-\infty}^{\infty} \int_{0}^{\Delta t} p(x-v s, y) d s d x d y
$$

We next show how $C_{\Lambda}(\Delta x, \Delta y)$ is related to

$$
c_{z}(h)=\operatorname{Cov}[z(t), z(t+h)]
$$


First. we consider

$$
c_{x}(\Delta x, \Delta y)=\operatorname{Cov}[x(x, y, t), x(x+\Delta x, y+\Delta y, t+\Delta t)]
$$

$\left(C_{x}\right.$ will in fact turn out to be independent of $\Delta t$, as well as of $x, y$ and t.) By definition, this is

$$
E\{[x(x, y, t)-E x(x, y, t)][x(x+\Delta x, y+\Delta y, t+\Delta t)-E x(x+\Delta x, y+\Delta y, t+\Delta t)]\} .
$$

Conditioning on $\Lambda(x, y)$, this can be written as the sum of two terms,

$$
\begin{aligned}
& E(E\{[x(x, y, t)-\Lambda(x, y)][x(x+\Delta x, y+\Delta y, t+\Delta t)-\Lambda(x+\Delta x, y+\Delta y)] \mid \Lambda\}) \\
& \quad+\operatorname{Cov}\{E[x(x, y, t) \mid \Lambda], E[x(x+\Delta x, y+\Delta y, t+\Delta t) \mid \Lambda]\} .
\end{aligned}
$$

As $E[x(x, y, t) \mid \Lambda]=\Lambda(x, y)$, the second term in Eq. (6) is just $c_{\Lambda}(\Delta x, \Delta y)$. In the first term, we can assume that the random variates $x(x, y, t)$ and $x(x+\Delta x$, $y+\Delta y, t+\Delta t$ ) are independent, given $\Lambda$, unless $\Delta x=\Delta y=\Delta t=0$. (That is, the deviation of $x$ from its conditional expected value $\Lambda$ at one point in space and time is independent of the deviation at a different point in space and/or at a different time.) When $\Delta x=\Delta y=\Delta t=0$, the first term in Eq. (6) is

$$
E\{\operatorname{Var}[x(x, y, t) \mid \Lambda]\}=E \Lambda(x, y)=m,
$$

using Eq. (2). Thus,

$$
C_{X}(\Delta x, \Delta y)= \begin{cases}C_{\Lambda}(0,0)+m & \Delta x=\Delta y=0 \\ C_{\Lambda}(\Delta x, \Delta y) & \text { otherwise. }\end{cases}
$$

Next we consider 


$$
\begin{aligned}
C_{I}(h)= & \operatorname{cov}[I(t), I(t+h)] \\
= & \iint_{-\infty}^{\infty} d x d y \int_{-\infty}^{\infty} \int_{t-\Delta t} d \xi d n \int_{t+h-\Delta t}^{t} d s \int_{t+h}^{t} d \tau(x, y) p(\xi, n) \\
& \quad \operatorname{cov}[x(v s-x,-y, s), x(v \tau-\xi,-\eta, \tau)] .
\end{aligned}
$$

Make the following changes of variables:

$$
\begin{aligned}
& Z=t-s \\
& r=x+v z \\
& \zeta=t+h-\tau \\
& \rho=x-\xi+v h-v \zeta \\
& \sigma=y \eta .
\end{aligned}
$$

The above ir tegral then becounes

$$
\begin{aligned}
\iint_{-\infty}^{\infty} d r d y \iint_{-\infty}^{\infty} d \rho d \sigma \int_{0}^{\Delta t} d z \int_{0}^{\Delta t} d \zeta p(r-v z, y) p(r-\rho+v h-v \zeta, y-\sigma) \\
\\
\operatorname{Cov}[x(v t-r,-y, t-z), x(v t-r+\rho,-y+\sigma, t+h-\zeta)] .
\end{aligned}
$$

The covariance is just $C_{X}(\hat{p}, \sigma)$, independent of all of the other integration variables. Define

$$
p_{v}(\alpha, \beta)=\int_{0}^{\Delta t} p(\alpha-v \tau, \beta) d \tau .
$$

Then after performing the integrals over $z$ and $\zeta$, what remains is

$$
\iint_{-\infty}^{\infty} d \rho d \sigma c_{X}(\rho, \sigma) \iint_{-\infty}^{\infty} d r d y p_{V}(r, y) p_{V}(r+y h-\rho, y-\sigma) .
$$


Define the inner integral as $c_{p}(v h-\rho,-\sigma)$, which leads finally to

$$
\begin{aligned}
C_{I}(h) & =\int_{-\infty}^{\infty} \int_{X}(\rho, \sigma) C_{p}(v h-\rho,-\sigma) d \rho d \sigma \\
& =\left(C_{X}^{*} C_{p}\right)(v h, 0),
\end{aligned}
$$

where * denotes the convolution of two functions,

$$
\left(f^{*} g\right)(x, y)=\iint f(\zeta, n) g(x-\zeta, y-\eta) d \zeta d \eta \text {. }
$$

Thus, the covariance function of $I$ is equal to the covariance function of $x$ (which in turn was essentially the covariance function of $\Lambda$, differing from the latter only at $h=0$, and this makes no difference in the integral) convolved with a function which depends on the point spread of the instruments.

Finally, from Eqs. (3) and (4), assuming that the signal from the ground, $I(t)$, the background signal $B(t)$, and the measurement noise $n(t)$ are all uncorrelated, we get

$$
\begin{aligned}
C_{Z}(h) & =\operatorname{Cov}[Z(t), Z(t+h)] \\
& =\left\{\begin{array}{l}
\varepsilon^{2}\left[\left(C_{\Lambda} * C_{p}\right)(v h, 0)+C_{B}(h)\right], h \neq 0 \\
\varepsilon^{2}\left[\left(C_{\Lambda} * C_{p}\right)(v h, 0)+C_{B}(h)\right]+E(\operatorname{Var} n(t)), h=0 .
\end{array}\right.
\end{aligned}
$$

Some examples will make the nature of the terms in Eq. (10) clearer. The function $C_{p}$ arose as a convolution-like integral,

$$
c_{p}(x, y)=\iint_{-\infty}^{\infty} p_{v}(\zeta, n) p_{v}(\zeta+x, n+y) d \zeta d n,
$$


where

$$
p_{v}(x, y)=\int_{0}^{\Delta t} p(x-v t, y) d t .
$$

Several models for the point-spread function $p$ have been proposed. Two are based on simple geometrical considerations (Ref. 3). They are not ton different from one which appears in Ref. 4. Figures 1 and 2 are based on the "elementary rod" model in Ref. 3, normalized as

$$
\begin{aligned}
p(x, y) & =p^{\prime}(I) \\
& =\exp \left(-\mu \sqrt{\left.h^{2}+I^{2}\right)} \cdot \frac{h}{4 \pi\left(h^{2}+r^{2}\right)^{3 / 2}}\right.
\end{aligned}
$$

In Eq. (11), $I^{2}=x^{2}+y^{2}, h$ is the altitude of the aircraft and $\mu$ is a frequency-dependent linear coefficient. Figure la is a plot of $p(x, 0)=p^{*}(x)$ for $h=125 \mathrm{~m}$ and $\mu=0.005 \mathrm{~m}^{-1}$. Figure lo is a plot of $p_{v}(x, 0)$ for $\Delta t=$ 1 second, $v=120 \mathrm{mph}$. The second $x$-axis converts units of distance to units of time at the given velocity. Figure lc shows the corresponding function $c_{p}(x, 0)$. Figures $2 a$ and $2 b$ are for a velocity of $70 \mathrm{mph}$, more typical of helicopter speeds.

In general, we can expect any signal, such as $A$ or $B$, to have what we will call both a "continuous" cumponent ard a noise component. By this we mean that the covariance function has a discontinuity at the origin, for example,

$$
c_{\Lambda}(0,0) \notin \lim _{\Delta x, \Delta y \rightarrow 0} c_{\Lambda}(\Delta x, \Delta y)
$$

$c_{\Lambda}(0,0)$ is the total variance of $\Lambda$. In the case of a geological process, such as $\Lambda$, it may be hard to visualize a sharp discontinuity in any realization $\lambda$, but it is often the case that estimates of $C_{\Lambda}(\Delta x, \Delta y)$ strongly suggest that Eq. (12) holds. The reason for this is probably that $\lambda$ has not been sampled finely enough. The small-scale structure in $\lambda$, if it were studied, might lead to a smooth extrapolation of $C_{\Lambda}$ to zero (apart from negligible measurement error), but when samples are available only at greater distances, the 
estimated autocovariance function appears to have a large discontinuity or "nugget" at the origin. In tile case of the background signal, this discontinvity is due to the random Poisson nature of the signal. In any case, their covariance functions have in general a shape such as shown in Fig. 3, where the central spike has zero width in the case of $C_{B}$ or small but finite width in the case of $c_{\Lambda}$. The "continuous" component of $\Lambda$ corresponds to the remaining positive portion of the function,

$$
C_{\Lambda}(\Delta x, \Delta y)=C_{\Lambda}(0) \quad \operatorname{Corr}[\Lambda(x, y), \Lambda(x+\Delta x, y+\Delta y)]
$$

and is positive at distances over which the $\Lambda$ process is positively correlated. That is, nonzero correlation over short distances is taken to represent some degree of continuity in the unaerlying process.

The following data examples are taken from the single record reduced count rates for the Rawlins quadrangle, a segment which was flown by helicopter over the tertiary Browns Park formation (TBP). These reduced count rates are obtained after considerable manipulation of the raw window count rates, the quantities that might reasonably be modeled by $z(t)$ in Eq. (4). However, perhaps because the reduction procedures are linear operations, even these data show the structure anticipated in Eq. (10).

The thallium record (Fig. 4a) has little structure apart from some short trends over distances on the order of two kilmeters. The total variance of this segment $\left[C_{Z}(0)\right.$ in the notation of Eq. (J 1$\left.)\right]$ is 47.3 , while the next points in the autocovariance estimate ( $F i g$. 4b) drop down to something on the order of 13 to 16 . Thus, the noise term [E(Var $\eta)$ in Eq. (10)] has a magnitude of about 32, and the signal in Fig. $4 \mathrm{a}$ is more than two-thirds noise, which certainly agrees with the visual impression. Part of this is undoubtedly due to a pure noise component in $C_{B}(h)$; that is, we suspect that the background signal is partially uncorrelated from second to second, although in part it comes also from highly continuous sources, such as the "bismuth-air" signal. The continuous part of the covariance, the $c_{\Lambda}{ }^{*} C_{p}$ term (which is always continuous as $h \rightarrow 0$ even though $c_{\Lambda}$ may not be continuous by itself) and the continuous part of $C_{B}(h)$, are reflected by the decline of the sample autocovariance function from $13-16$ at $\mathrm{h} \sim \mathrm{O}$ to zero at about $2.0 \mathrm{~km}$. As $2.0 \mathrm{~km}$ is considerably greater tran the width of $C_{p}$, the positive correlation out to this distance must the ascribed either to some underlying correlation in $\Delta$ 
out to distances of $1.5 \mathrm{~km}$ or greater, or to the continuous (correlated) part of the background signal, or both. (The effect of convolving $c_{p}$ with $c_{\Lambda}$ is to broaden $C_{\Lambda}$ by about the haif-width of $c_{p}$, which was not more than $0.5 \mathrm{~km}$; for this reason, if $C_{\Lambda} * C_{p}$ is positive out to $2.0 \mathrm{~km}, c_{\Lambda}$ must have been positive out to at least $1.5 \mathrm{~km}$.) At the moment we have no way of estimating the relative contributions of the two terms to the continuous part of $C_{Z}$. For this purpose, it will be necessary to do some analyses of the "bismuth-air" and "cosmic" signals, as well as to investigat " the details of Geometrics' instrument and aircraft correctinn prncedures.

The reduced potassium record for the same segment is shown in Fig. 5a. The visual impression given by these data is that the signal-to-noise ratio is higher than in Fig. 4a, and this is confirmed by Fig. 5b, which shows that $\lim _{h \rightarrow 0} C_{Z}(h)$ is about 600 , more thari $80 \%$ of the total variance $c_{Z}(0)$. The potassium signal is also apparently nositively correlated out to at least 3.0 $\mathrm{km}$.

The reduced bismuth signal is interesting because of the sharp jump at about $9.0 \mathrm{~km}$ (Fig. 6a). This presumably reflects a point anomaly on the ground, and the effect of such a discontinuity is to add a significant contribution to the underlying discontinuous part of $c_{\Lambda}$. When this spike at the origin is convolved with $C_{p}$ the result has the shape of $C_{p}$, and this is clearly visible as a hump in Fig. 6b. The noise component in this case is about 30.0 (about half of the total). From about 34.0, sample autocovarience function decreases to about 23.0 , at $0.5 \mathrm{~km}$, with a shape like that seen in Fig. 2b. The continuous part of $C_{Z}(h)$ extends farther, to perhaps $3.5 \mathrm{~km}$. This extension, again, comes from the continuous part of $C_{\Lambda}$ convolved ith $C_{p}$ and/or the continuous part of $C_{B}$. 


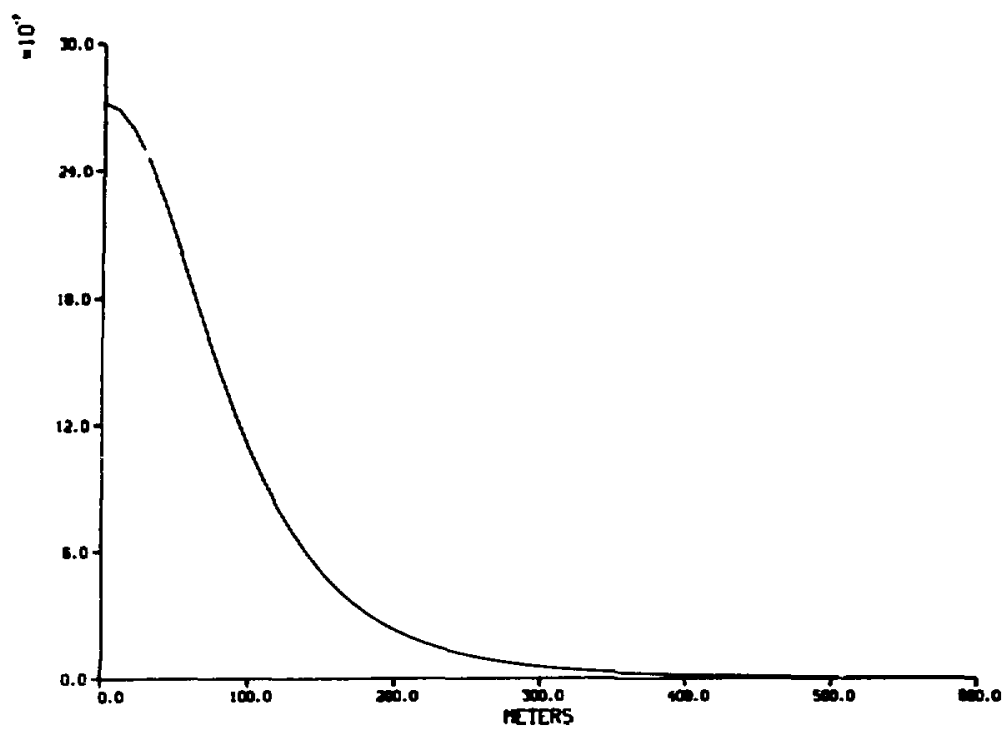

Fig. la. One model for an instrumental pointspread function.

ONE-SECOND POINT-SPREAD FUNCTION

VELOCITY - 120 H.P.H.

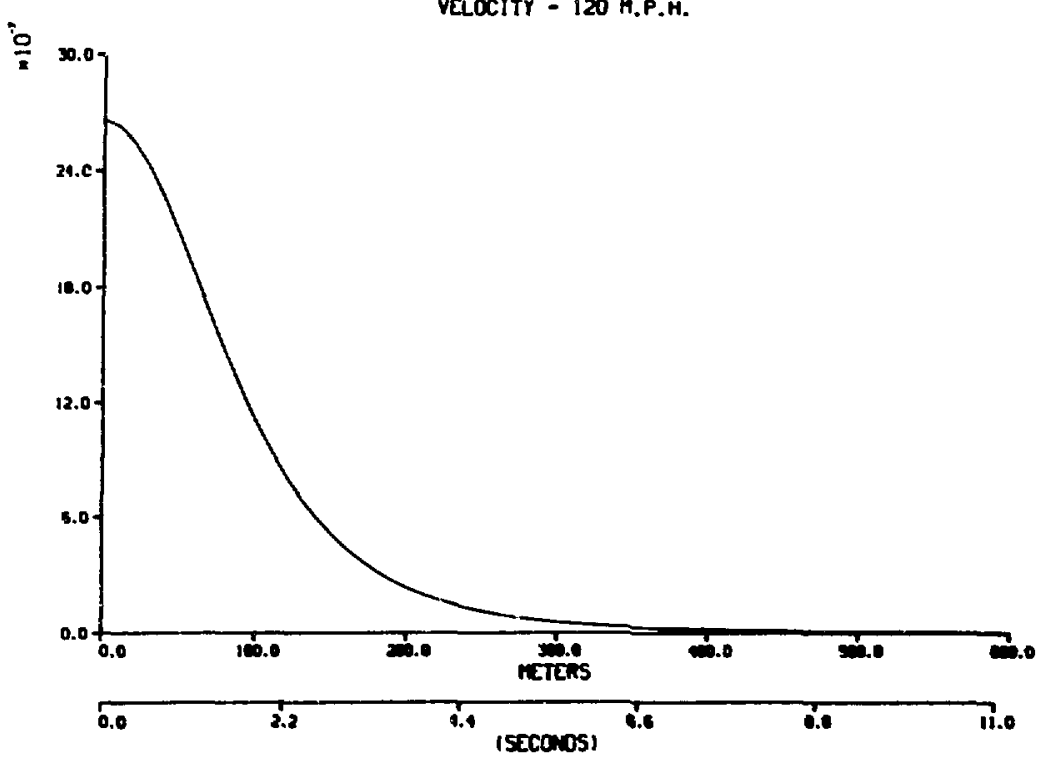

Fig. Ib. Widening of point-spread function due to integration over one second at $120 \mathrm{mph}$. 


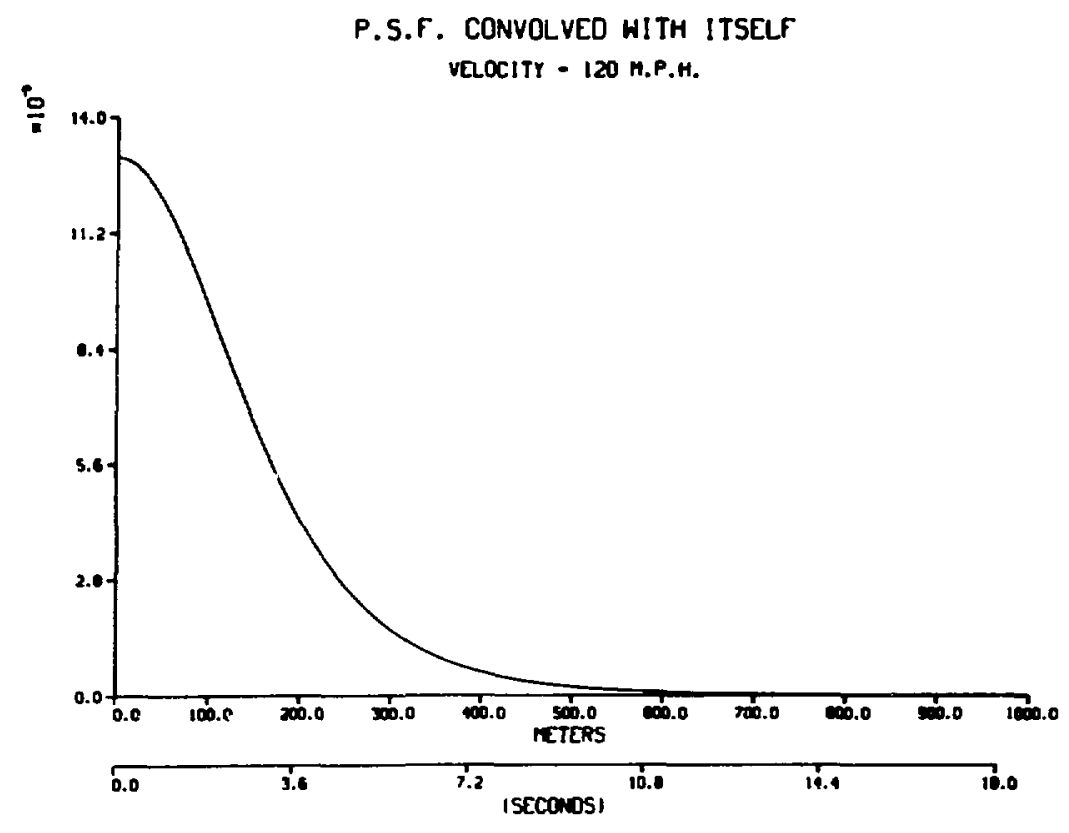

Fig. 1c. Convolution of the point-spread function with itself (120 $\mathrm{mph})$.

ONE-SECOND POINT-SPREAD FUNCTION

VELOCITY - 70 M.P.H.

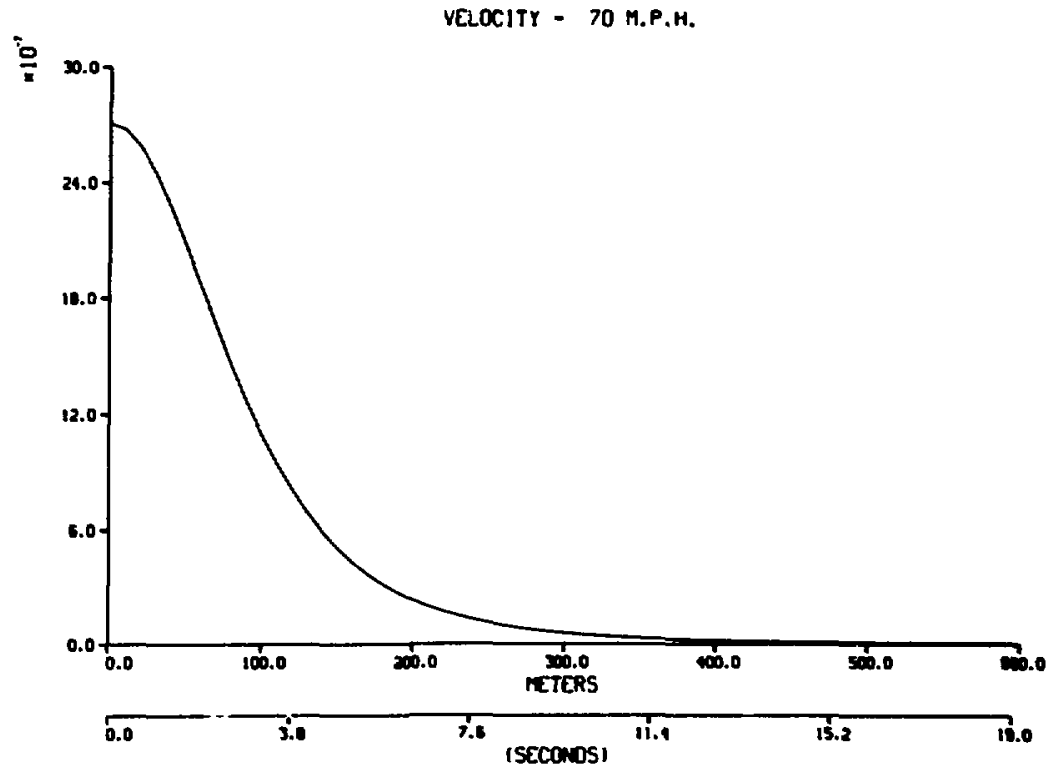

Fig. 2a. Widening of point-spread function at $70 \mathrm{mph}$. 


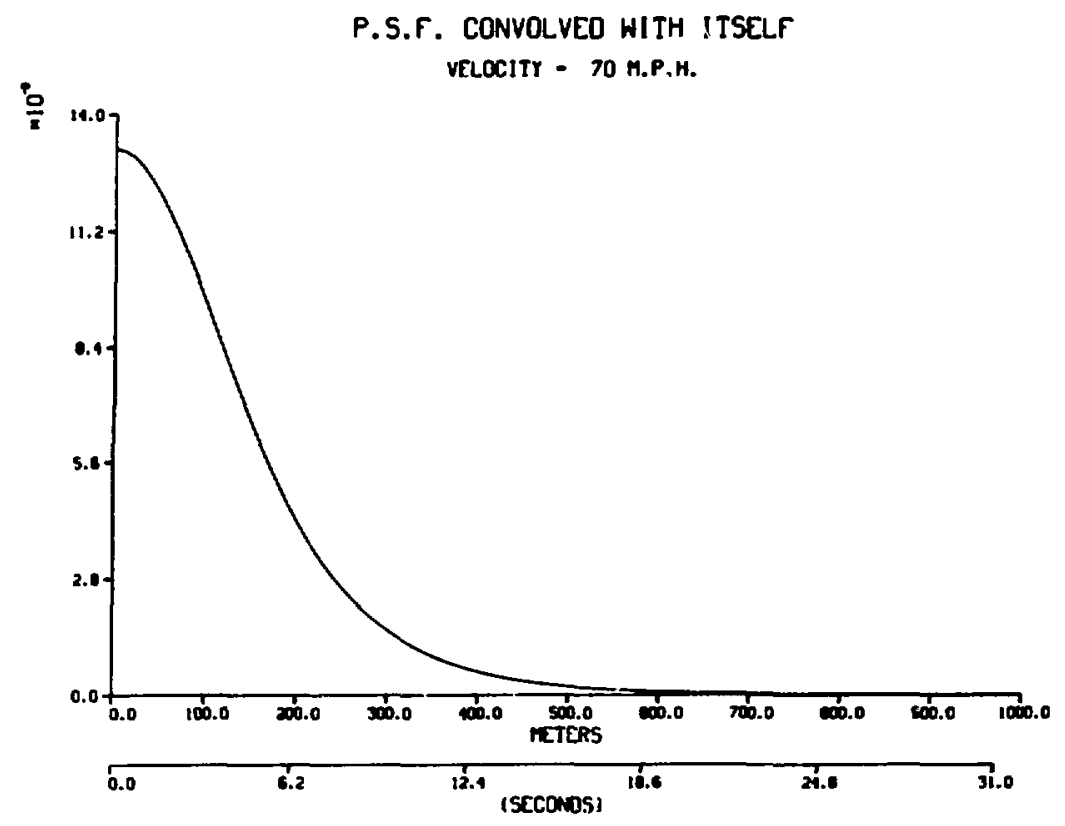

Fig. 2b. Convolution of point-spread function with itself (70 $\mathrm{mph})$.

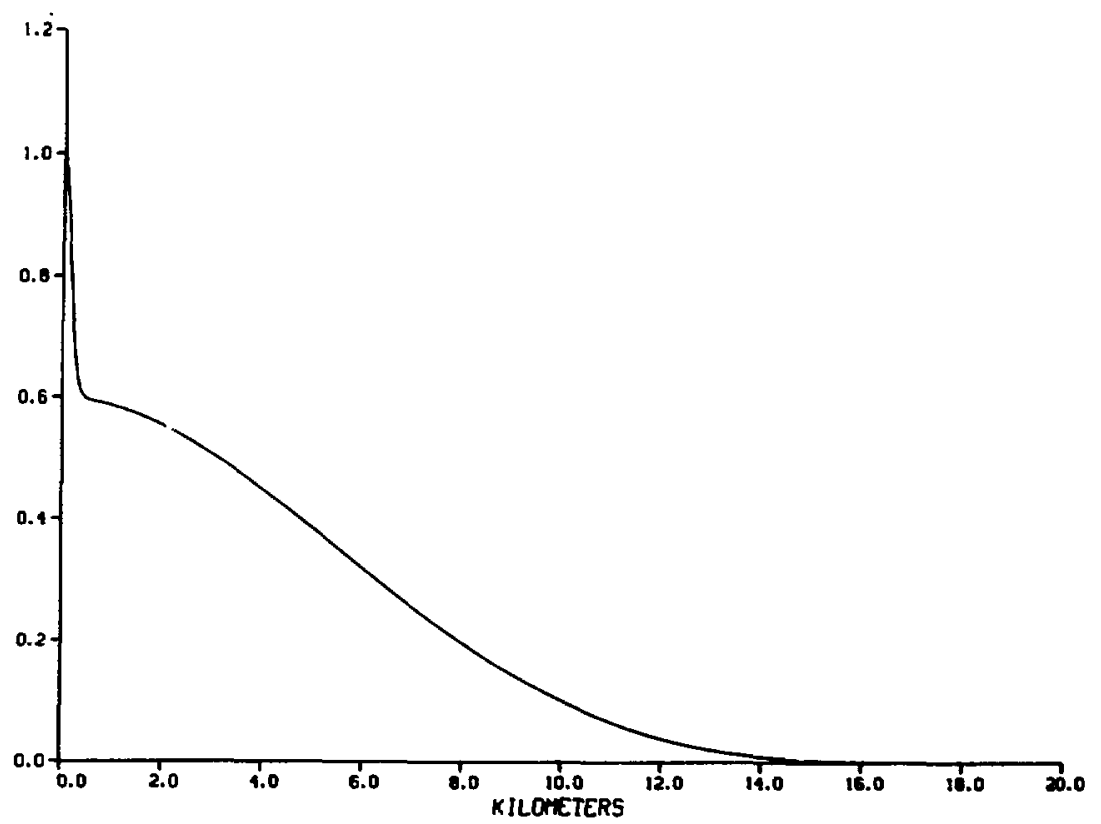

Fig. 3. Typical shape for autocovariance function of correlated, noisy observations. 
THRLLIUM LINE NUMBER $220 \quad$ I TBP I
IAVERAGE VELOCITY - 65.43 H.P.H.I

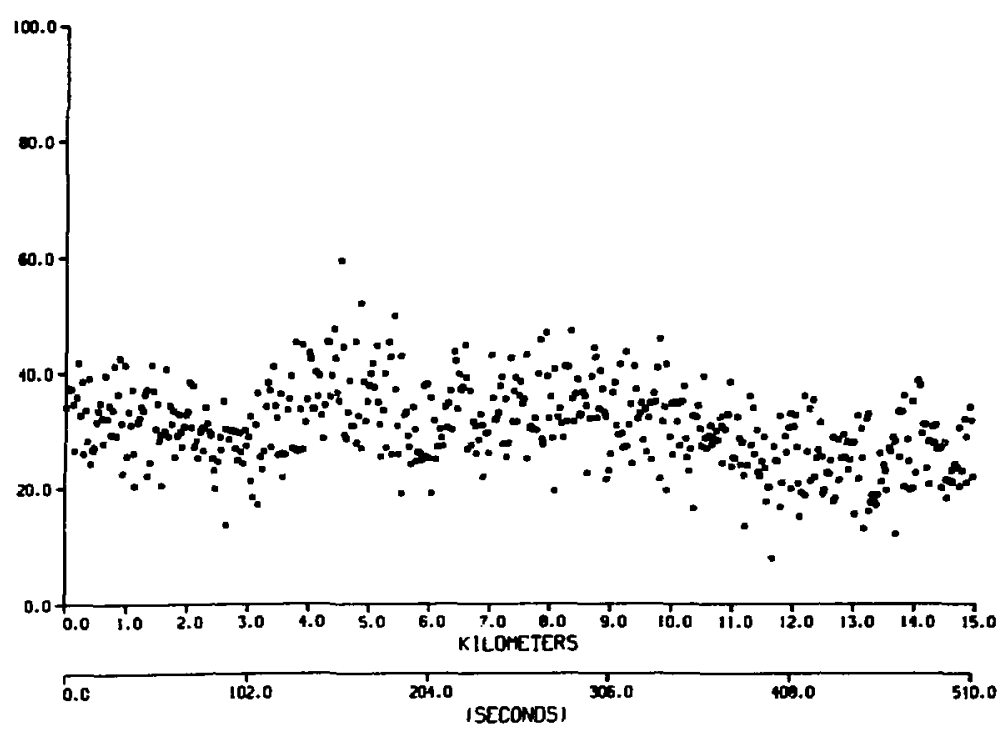

Fig. 4a. Single record reduced count data for $208 \mathrm{~T} \ell$.
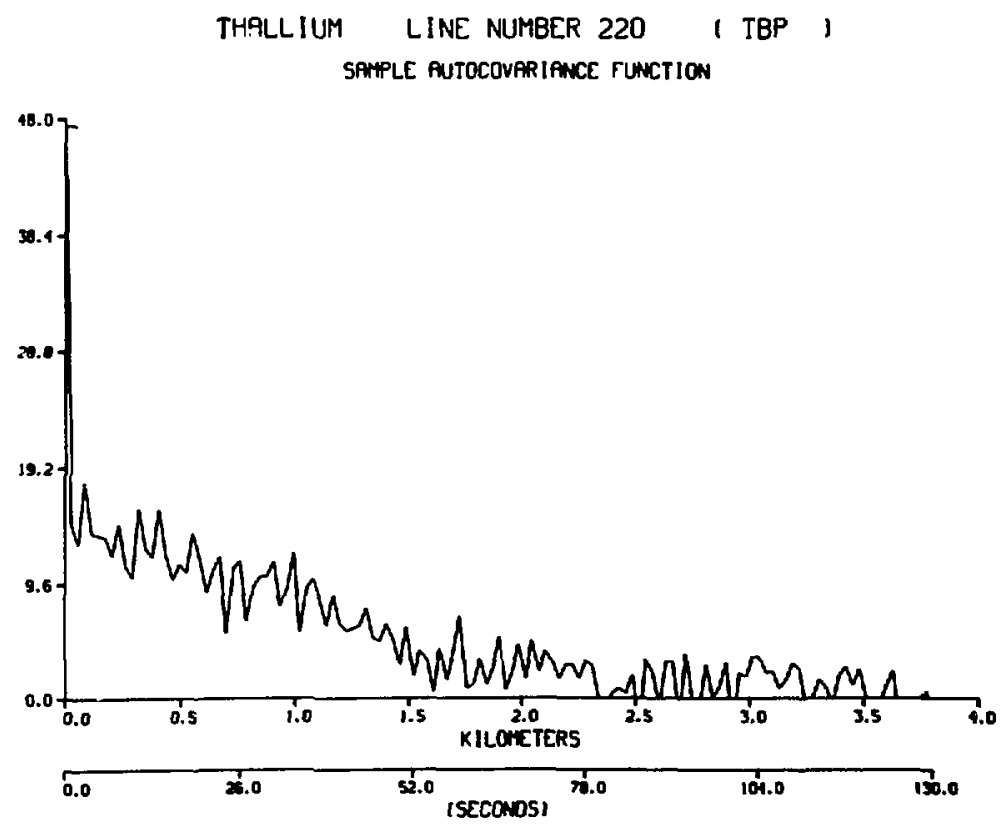

Fig. 4b. Sample autocovariance function for $20 \mathrm{~B}$ T. 
POTASSIUM LINE NUMBER 220 ( TBP )

(AVERAGE VELOCITY - 65.43 M.P.H.)

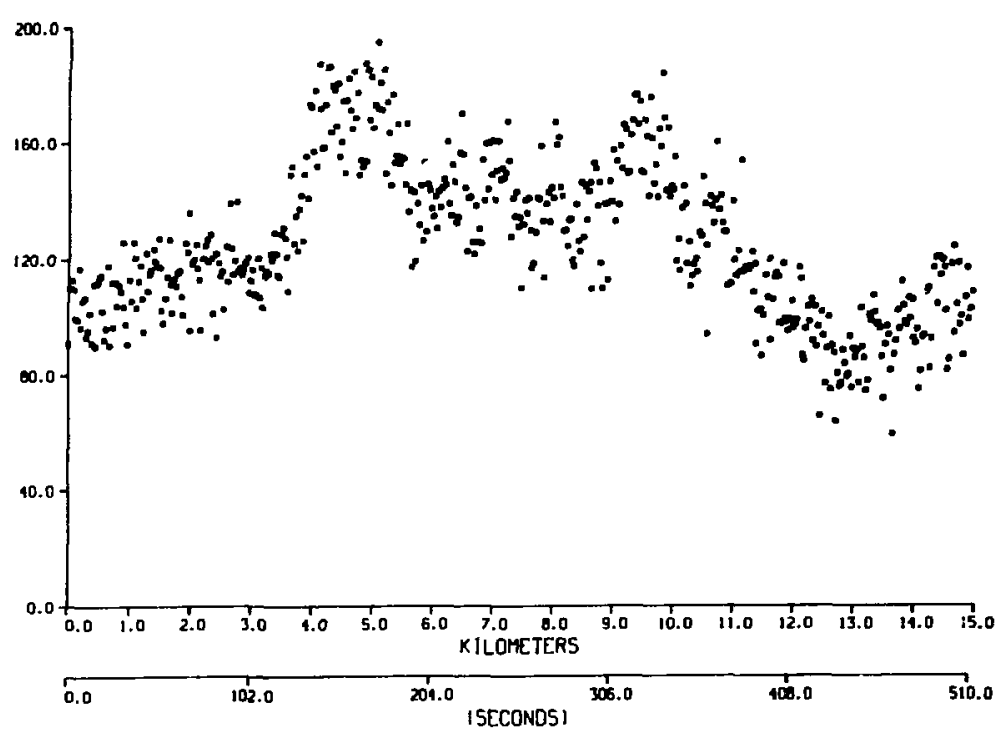

Fig. 5a. Single record reduced count data for ${ }^{40} \mathrm{~K}$.

POTASSIUM LINE NUMBER $220 \quad 1$ TBP ।

SATPLE RUTOCONARIANCE FUNCTION

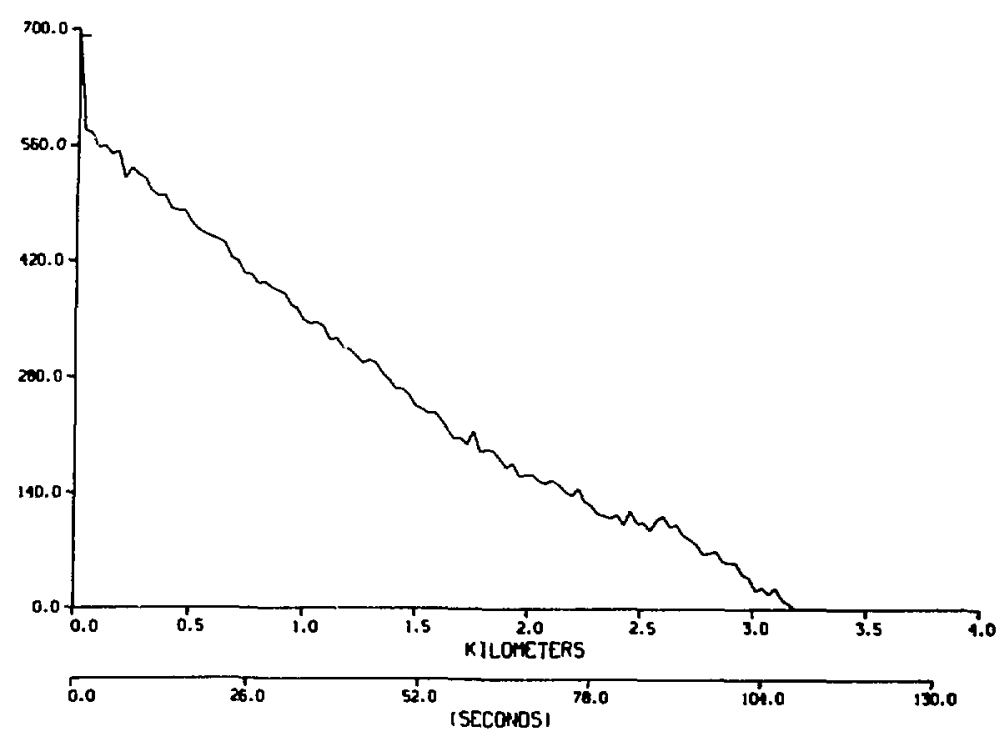

Fig. 5b. Sample autocovariance function for ${ }^{40} \mathrm{~K}$ data. 
BISMUTH LINE NUMBER 220 । TBP J

(AVERAGE VELOCITY - 65.43 M.P.H.)

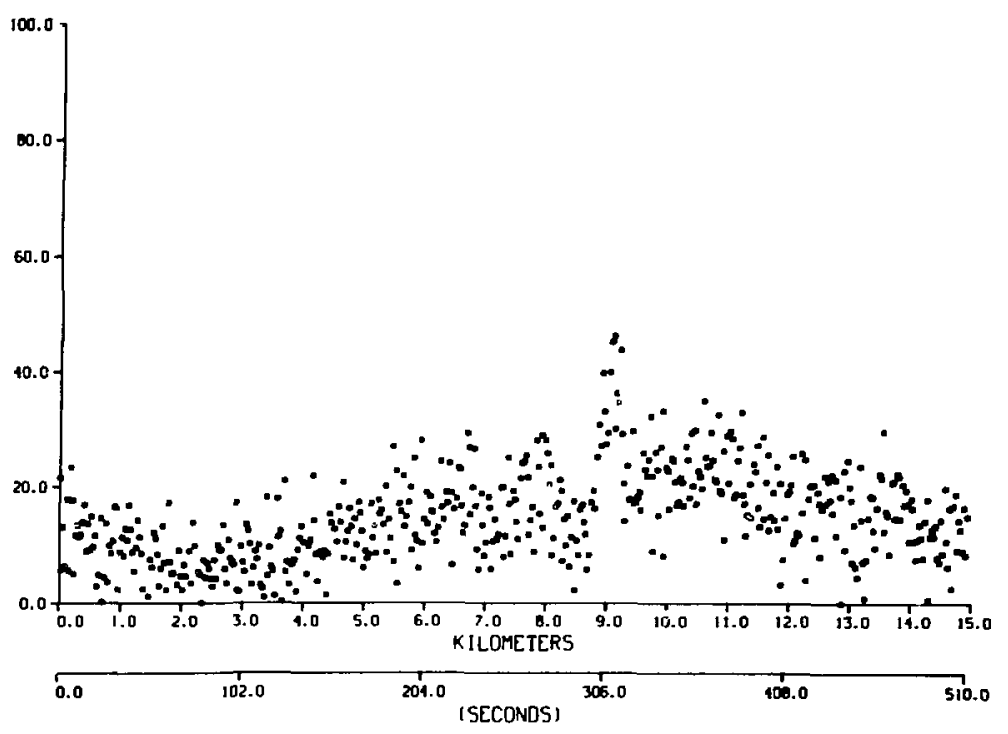

Fig. 6a. Single record reduced count data for $214 \mathrm{Bi}$.

BISMUTH LINE NUMBER 220 । TBP ।
SAMPLE AUTOCOVARIANCE RUNCTION

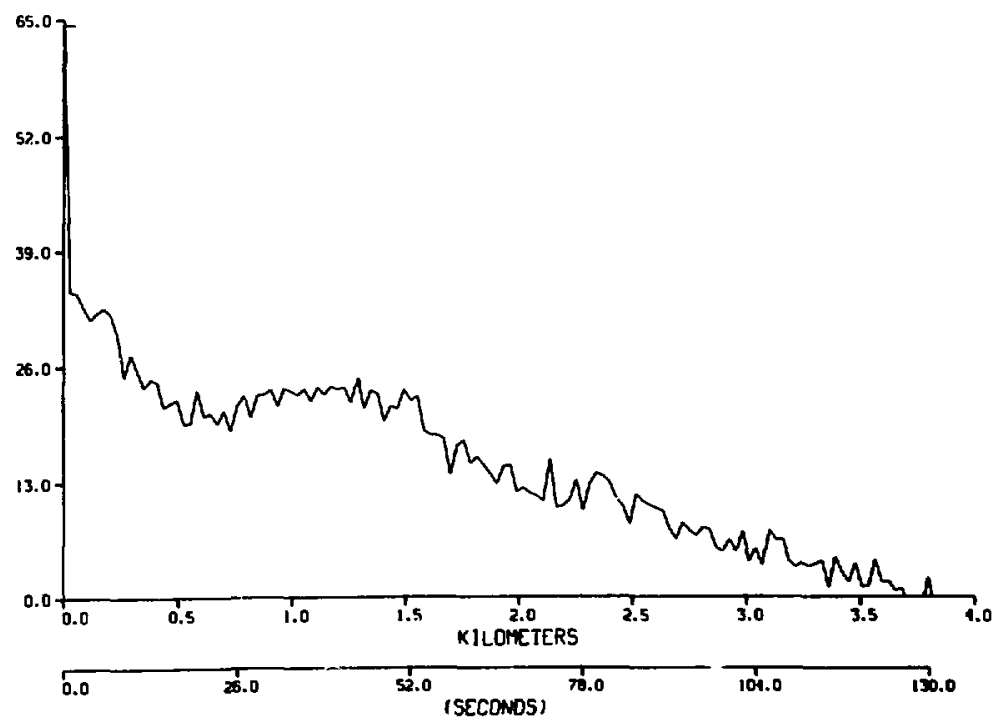

Fig. 6b. Sample autocovariance function for $214 \mathrm{Bi}$. 


\section{B. Other Descriptions of Serial Properties}

There are a number of functions which are related to the autocovariance function $c_{Z}(h)$. One of the best known is the power spectrum,

$$
S_{Z}(\omega)=\int_{-\infty}^{\infty} e^{-i 2 \pi \omega h} c_{Z}(h) d h
$$

(also called the spectral density function). $S_{Z}(\omega)$ is estimated by the periodogram

$$
I(\omega)=\frac{\Delta h}{N}\left|\sum_{n=1}^{N} z(n \Delta h) e^{-i 2 \pi \omega n}\right|^{2},
$$

assuming that the process $Z(h)$ has been sampled at regular intervals $\Delta h$. It can be shown that

$$
I(\omega)=\Delta h \sum_{k=-\infty}^{\infty} e^{-i 2 \pi \omega k} c_{Z}(k \Delta h),
$$

in immediate analogy with Eq. (13), where $c_{Z}(k \Delta h)$ is the sample autocovariance function

$$
c_{Z}(k \Delta h)= \begin{cases}\frac{1}{N} \sum_{n=1}^{N-|k|}[(Z(n \Delta h)-\bar{Z}][Z((n+|k|) \Delta h)-\bar{Z}] & 0 \leq|k|<N \\ 0 & |k| \geq N .\end{cases}
$$

(These are the functions plotted in Figs. $4 b, 5 b$ and $6 b$, estimating $C_{Z}(h)$. ) Under the Fourier transform of Eq. (13), Eq. (10) becomes

$$
S_{Z}(w)=\varepsilon^{2}\left[S_{\Lambda}(w) \cdot S_{p}(w)+S_{B}(w)\right]+E(\operatorname{Var} n) .
$$

$S_{p}(w)$ [the transform of $C_{p}(x, 0)$ ] is shown in Fig. 7. Figure 8 is the periodogram of the data in Figure 4a. The transforms of $C_{\Lambda}$ and $C_{B}$ are much narrower than $S_{p}$, so the periodogram falls off rapidly to about 0.5 cycles 
per $\mathrm{km}$, where it is virtually down to the noise level (the constant $E($ Var $\eta$ ) times $\Delta \mathrm{h}$; the product is slightly less than 1 ).

Another function, which will be useful in establishing a criterion for the minimum bias width in tne maximum variance segments algorithm, is an integral of $c_{Z}(h)$ called the variance-length curve,

$$
v_{Z}(L)=C_{Z}(0)-\frac{L}{L^{2}} \int_{0}^{L}(L-h) C_{Z}(h) d h .
$$

It can be shown that $V_{Z}(L)$ is the expected variance of $Z$ within a segment of length $L$,

$$
v_{Z}(L)=E\left\{\frac{1}{[} \int_{0}^{L}\left[Z(t)-m_{L}\right]^{2} d t\right\},
$$

where

$$
m_{L}=E\left[\frac{1}{L} \int_{0}^{L} Z(t) d t\right] .
$$

If $Z(t)$ were a pure noise process, $V_{Z}(L)$ would be constant for all L. However, when $Z$ is positively correlated over short distances, $V_{Z}(L)$ is an increasing function of $L$, where $\lim _{L \rightarrow 0} V_{Z}(L)$ is the noise level (the discontinuous part of $Z$ ) and $\lim _{L \rightarrow \infty} v_{Z}(L)=\operatorname{Var} Z$.

The variogram

$$
\gamma_{Z}(h)=\frac{1}{2} \operatorname{Var}[z(t+h)-z(t)]
$$

can be defined even if $Z$ is not itself stationary, but does have stationary increments. When $Z$ is stationary, then

$$
r_{Z}(h)=c_{Z}(0)-c_{Z}(h)
$$

Figure 9 is the variogram for the data in Fig. 5, showing again the hump, now inverted, which arises from convolving $C_{p}$ with the discontinuous part of $\mathrm{C}_{\Lambda}$. Fig. 9 is not exactly $\mathrm{Fig}$. $5 \mathrm{~b}$ as inverted because the experimental variogram of Fig. 9 was computed as 


$$
\gamma_{z}(n \Delta h)=\frac{1}{2(N-n)} \sum_{k=1}^{N-n}[z(k \Delta h)-z((k+n) \Delta h)]^{2},
$$

which is not quite $c_{Z}(0)-c_{Z}(n \Delta h)$.

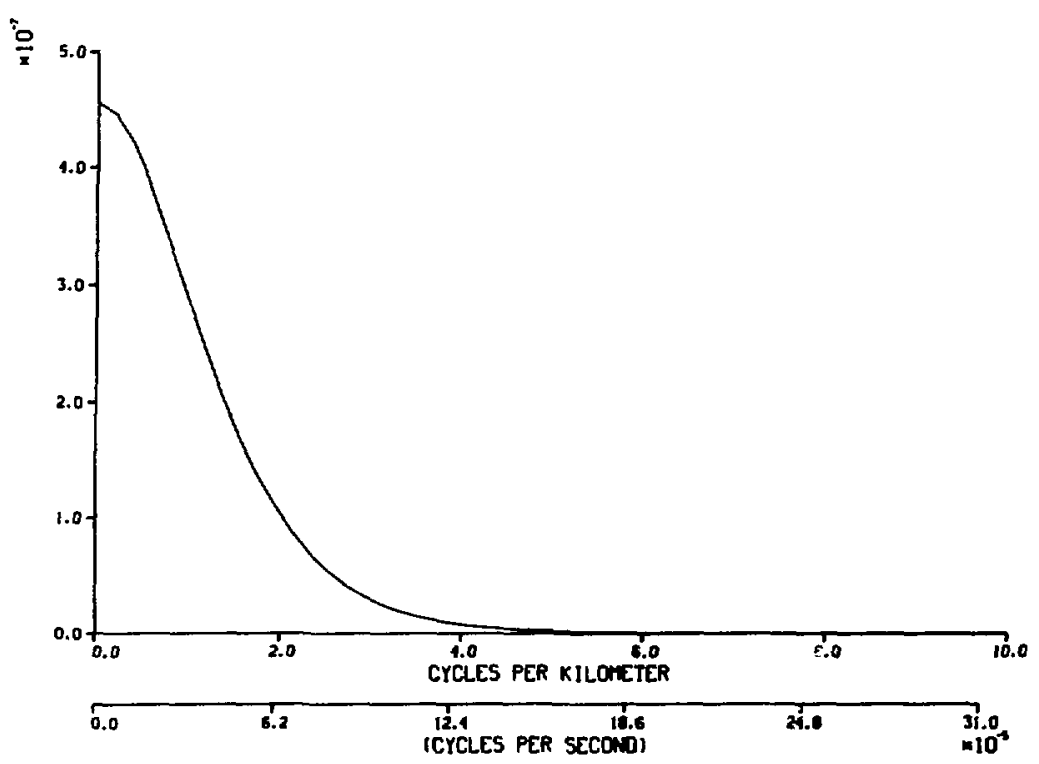

Fig. 7. Fourier transform of convolution of point-spread function with itself (70 mph). 
THRLLIUM LINE NUMBER 220 I TBP । PERIODOGRAM

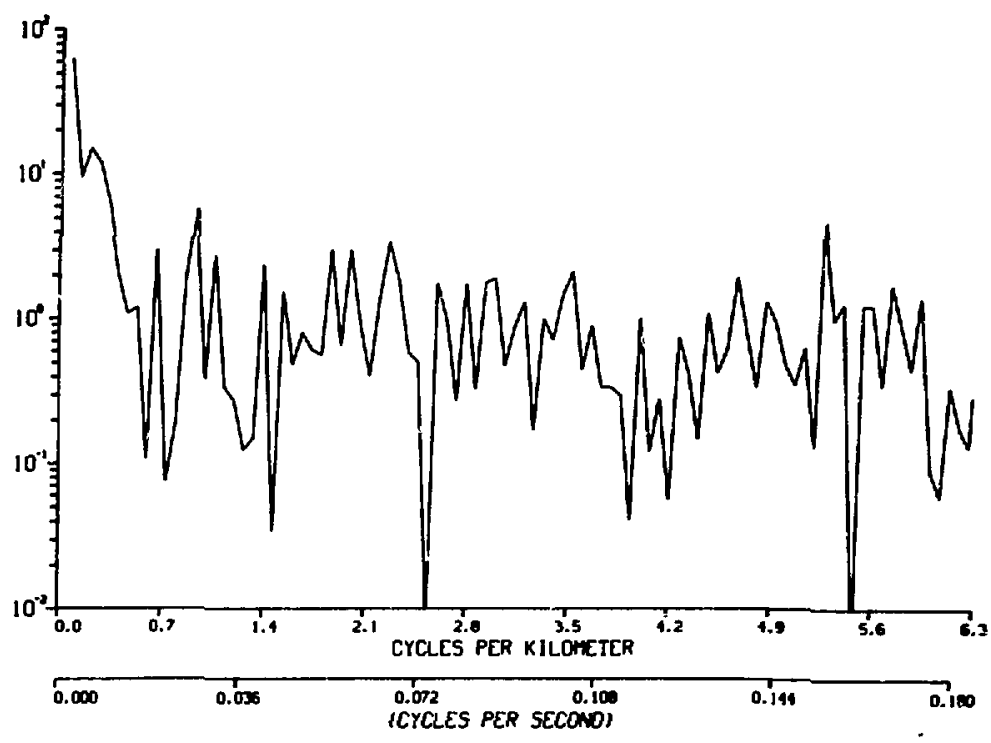

Fig. 8. Periodogram of ${ }^{208}$ T $\ell$ data. :

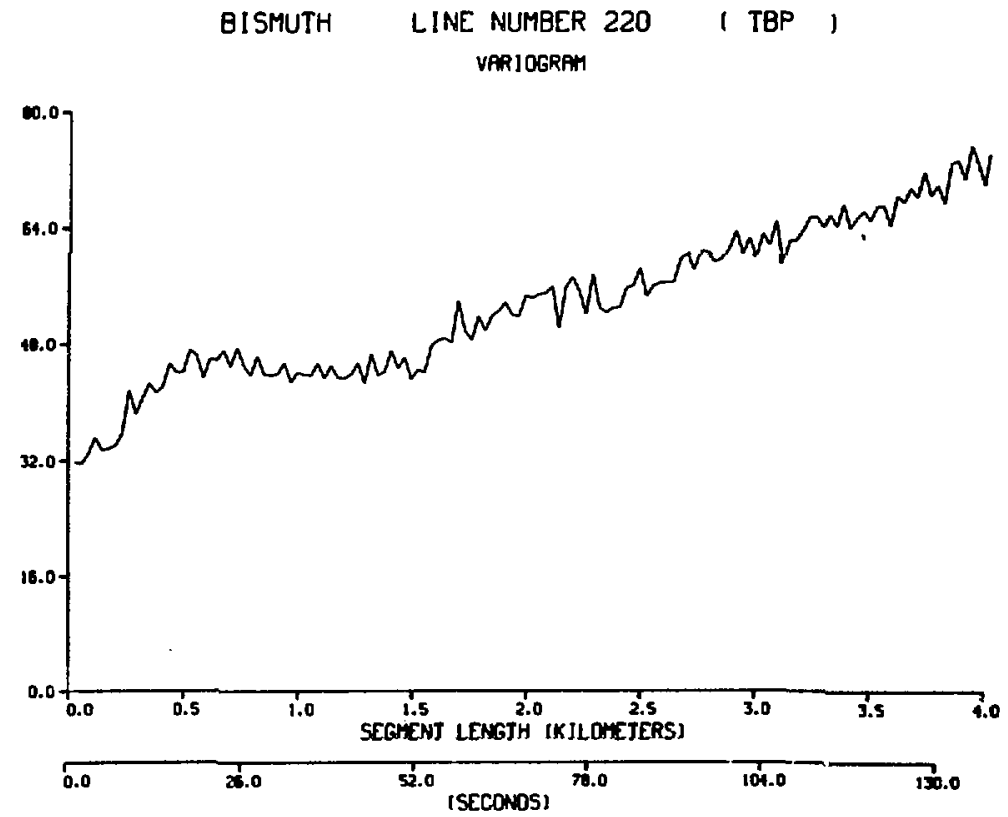

Fig. 9. Experimental variogram of ${ }^{214} \mathrm{Bi}$ data. 


\section{The Effect of a Moving Average}

One method which is popular for increasing the signal-to-noise ratio in a segment such as shown in Fig. $4 a$ is the use of a seven-point (sometimes five-point) moving average. This is equivalent to convolving $z(t)$ with a rectangular window

$$
R(t)= \begin{cases}1 & -a \leq t \leq a \\ 0 & \text { otherwise. }\end{cases}
$$

For the data in Fig. 4a, a is approximately $0.1 \mathrm{~km}$. The result of the convolution is shown in Fig. 10a. Indeed, the noise has been much reduced. Fig. $10 \mathrm{~b}$ is the corresponding sample autocovariance function, which is given by

$$
c_{Z}(h) * c_{R}(h)
$$

where $C_{R}(h)$ is the convolution of $R$ with itself, a triangular function of $h$ whose half-width is about $0.2 \mathrm{~km}$. The triangle is seen out to about $0.2 \mathrm{~km}$ in Fig. 10b, the result of convolution with the discuntinuous part of $c_{Z}(h)$. The remainder of the autocovariance function is smoothed and broadened slightly by the convolution (for example, Fig. 4b).

However, the moving average introduces some distracting artifacts which are very noticeable in Fig. 10a, specifically, small oscillations on the order of 2 to 3 cycles per kilometer. The reason for this is clearer if we consider the "frequency" domain. The Fourier transform of the convolution of $Z$ with $R$ is the product of the Fourier transforms of $Z$ and $R$. The transform of $R$ is shown in Fig. 1l. It is nearly zero for frequencies between about 4.5 and 5.25 cycles per kilometer, and thus multiplication with the transform of $Z$ suppresses these frequencies in the product. As a result, the lower frequencies, relatively unmodified (compare Fig. 12 with Fig. 8) become distractingly apparent in the original data. For this reason a filter with a smoother "roll-off" in both the spatial and frequency domains would be preferable. 
THALLIUM LINE NUMBER 220 I TBP ]

IAVERAGE VELOCITY - 65. 43 M.P.H.)

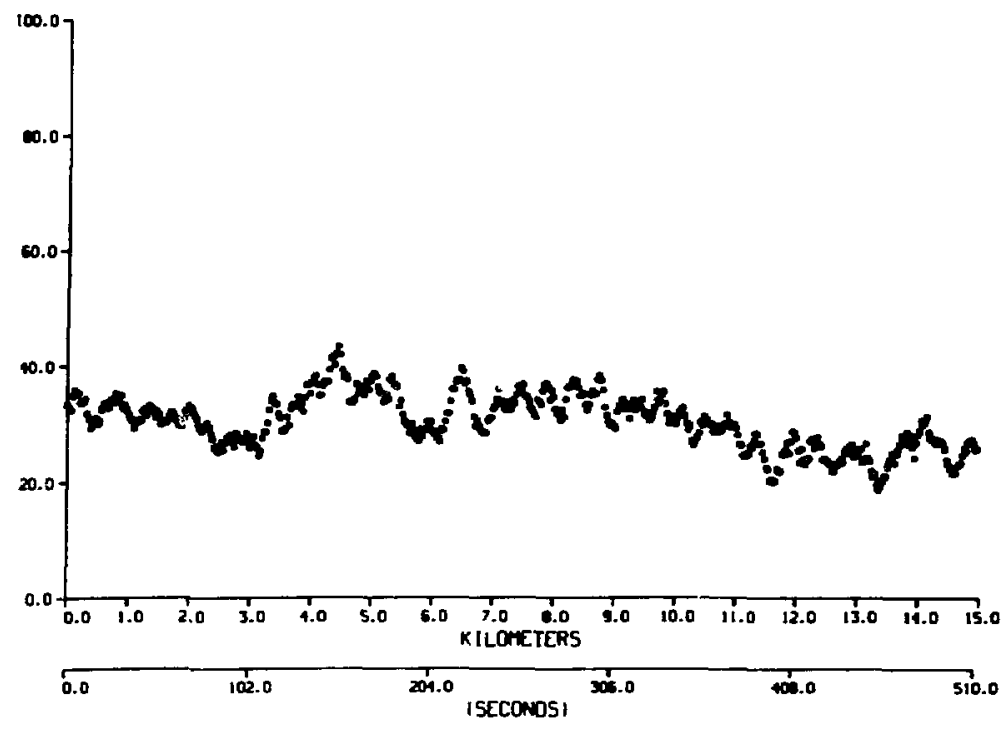

Fig. 10a. Result of applying a seven-point moying average to the ${ }^{208} \mathrm{~T} \ell$ data.
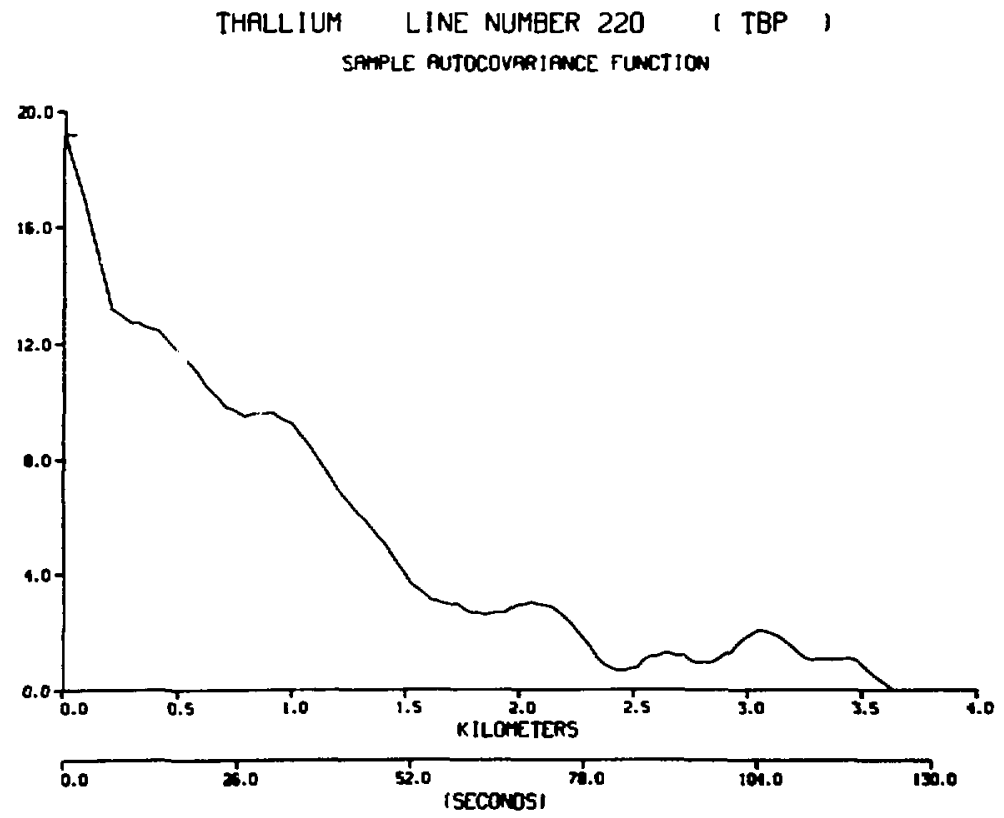

Fig. 10b. Sample autocovariance function of the averaged ${ }^{208} \mathrm{~T} \ell$ data. 
TRRNSFORM OF MOVING AVERAGE WINDOW

VELOCITY - 65.43 M.P.H.

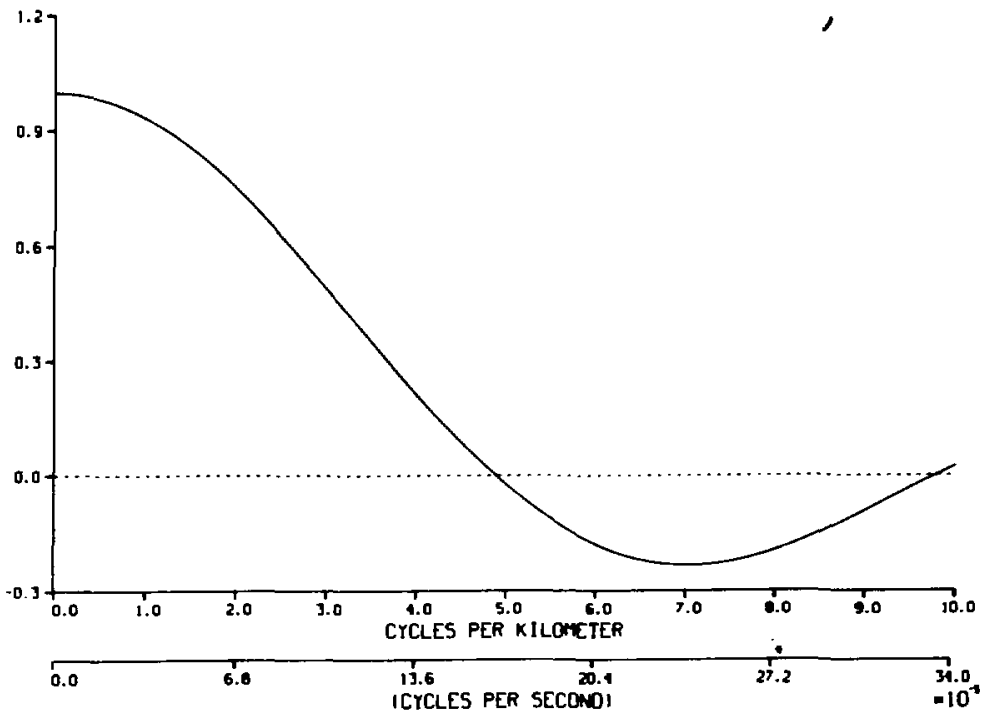

Fig. 11. Fourier transform of the seven-point moving average.

THRLLIUM LINE NUMBER 220 I TBP ,

PERIODOGRAN

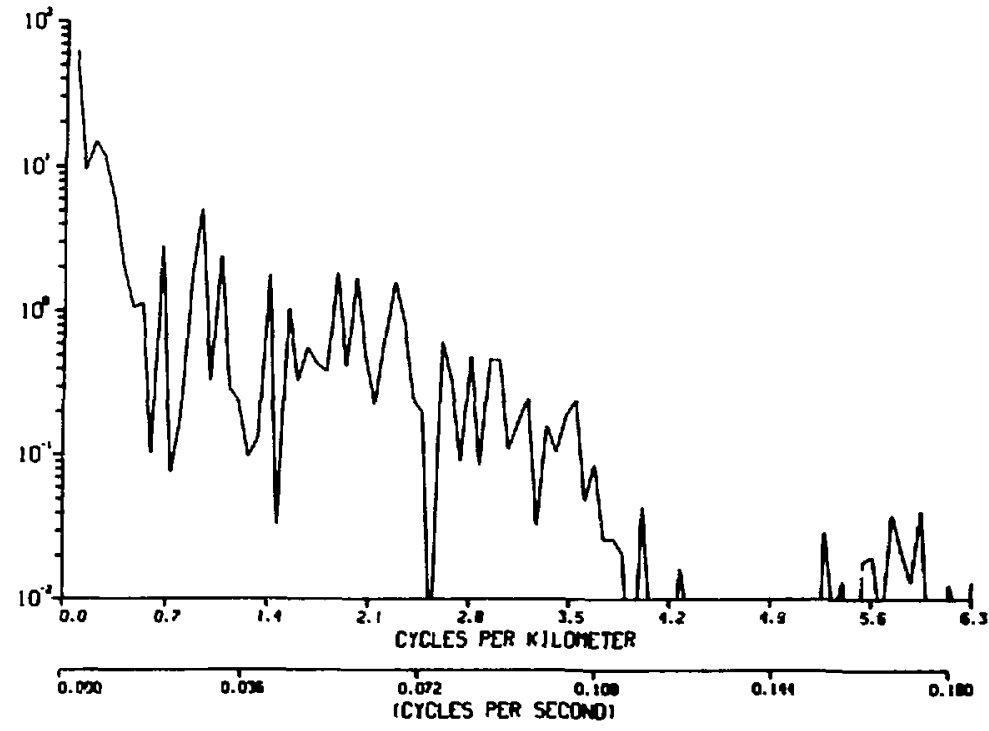

Fig. 12. Periodogram of the averaged ${ }^{208} \mathrm{~T} \ell$ data. 


\section{Selection of Minimum Length for "Maximum Variance Segments"}

Given that the current maximum variance segments algorithm almost irwariably produces segments of the present minimum length, $w$, the choice of $w$ becomes a matter of some interest. Fortunately, soine rational basis for this choice exists because of the correlation introduced into successive observations by the instruments. Each observation is a weighted average of the sinnal over a fairly large area on the ground beneath the plane. [The weighting function is the instrumental point spread function, one model of which was given in Ea. (11).] Even at ' $20 \mathrm{mph}$ the plane does not move very far in one second, compared to the area over which it is integrating. As a result, even if the ground signal is completely uncorrelated from one point to another, adjacent records are highly correlated, so that significant variation over short segments is attributable almost entirely to noise added by the recording procedure, not to variation in the signal. Therefore, short segments should be excluded from consideration by the maximum variance segments algorithm.

For present purposes, the correlation introduced by the instruments is most conveniently described by the variance-length curve, $v_{Z}(L)$, defined in Eqs. (16) and (17). Let us rewrite $C_{Z}(0)$ as the sum of an instrumental noise variance component, $V_{N}$, and an incoming signal variance component, $v_{S}$, and set

$$
C_{Z}(h)=v_{S} R(h), \quad h \neq 0,
$$

where $R(h)$ is the autocorrelation of two observations separated by a distance $h$ (in the absence of additional measurement error). Then Ea. (16) becomes

$$
V_{Z}(L)=V_{N}+V_{S}\left[1-\frac{L}{L^{2}} \int_{0}^{L}(L-h) R(h) d h\right] \text {, }
$$

and the fraction of the variance over a segment of length $L$ which can be ascribed to the signal (as opposed to the white noise added by the recording procedure) is

$$
\frac{V_{Z}(L)}{V_{N}}=1+\frac{V_{S}}{V_{N}}\left[1-\frac{2}{L^{2}} \int_{0}^{L}(L-h) R(h) d h\right] .
$$


Now, referring back to Eq. (10) we see that even if $C_{B}(h)$ were zero for $h=$ 0 (that is, the background signal were pure noise) and $c_{\Lambda}(\Delta x, \Delta y)=0$ for nonzero $\Delta x$ or $\Delta y$ (that is, the concentration of the source on the ground is uncorrelated from ore point to another), still the function $c_{Z}(h)$ would have nonzero values for $h \neq 0$, as it is proportional to $C_{p}$ (vh, 0 ), and $v_{Z}(L)$ would be an increasing function of $L$. This extreme case provides an upper bound on how fast $V_{Z}(L)$ can increase with $L$. Figure 13 shows plots of $V_{Z}(L) / V_{N}$ computed according to Eq. (2l), where $R(h)=c_{p}(v h, 0) / c_{p}(0,0)$ for three values of the overall signal-to-noise ratio $V_{S} / V_{N}$. Here $c_{p}$ is based on Eq. (11) with a velocity of $75 \mathrm{mph}$.

A reasonable way to select the minimum segment width, $w$, might be to require that $v_{Z}(w) / N_{N}>2$, because when $v_{Z}(L) / v_{N}<2$, the variability of a segment of length $L$ is more than $50 \%$ due to noise. Table $V$ gives the value of $w$, in meters, for which $v(w) N_{N} \sim 2$, read off the graphs of Fig. 13, for three values of the signal-to-noise ratio. In the third and fourth columns of Table $v$ these values are converted to seconds for observations from fixed-wing aircraft and helicopters.

In particular, a minimum segment width of five seconds for the example discussed in the section comparing the two maximum variance algorithms (which is based on helicopter dati, flown at about $75 \mathrm{mph}$ ) is certainly too small, as the signal-to-noise ratio is not greater than four.

If the signal on the ground enjoys some spatial autocorrelation, then the signal received by the instruments will be correlated out to larger distances and the variance-length curve, $v_{Z}(L)$, will rise more slowly than suggested by Fig. 13. Empirical variance-length curves suggest that this is indeed the case, and that it may take several kilometers for $V_{Z}(L) / V_{N}$ to rise above 2. However, this will be extremely variable from one string of data to another, and furthermore, it is precisely those cases where $V_{Z}(L) / V_{N}$ rises rapidly which are of interest. As computations based on the point-spread model give a lower bound on $L$ such that $V_{Z}(L) / V_{N}=2$, this seems to be a reasonable way to choose a lower bound on the length of segments to be considered. 
TABLE V

APPROXIMATE MINIMUM BIN WIDTH, SELECTEO BY REQUIRING

$$
v_{Z}(w) / V_{N}=2
$$

Signal-to-Noise

Ratio

2
3
4
Width

(meters)

460

300

240
Width (seconds)

At $125 \mathrm{mph}$

At $70 \mathrm{mph}$

$8-9$

$5-6$

4-5
14-15

$9-10$

$7-8$

The total signal plus noise is traditionally estimated by the total variance of the string of observations $z_{1}, z_{2}, \ldots, Z_{N}$ :

$$
v_{S}+v_{N}=\frac{1}{N} \sum_{i=1}^{N}\left(z_{i}-\bar{z}\right)^{2}
$$

Estimates of the signal variance, $V_{S}$, or the noise variance, $V_{N}$, are readily formed from plots such as Fig. $5 b$ [use $\lim c_{Z}(h)$ for $v_{S}$ ] or Fig. 9 [use $\lim _{h \rightarrow 0} \hat{\gamma}_{Z}(h)$ for $v_{N}$ ]. As a single computed indicator one might use

$$
v_{S}=\hat{C}_{Z}(\Delta h)=\frac{1}{N} \sum_{i=1}^{N-1}\left(z_{i}-\bar{Z}\right)\left(z_{j+1}-\bar{Z}\right)
$$

or

$$
V_{N}=\hat{\gamma}_{Z}(\Delta h)=\frac{1}{2(N-1)} \sum_{i=1}^{N}\left(z_{i}-Z_{i+1}\right)^{2}
$$

An estimate of the signal-to-noise ratio $V_{S} / V_{N}$ can be formed by any of the obvious combinations of eqs. $(22),(23)$ and (24). 
VARIANCE-LENGTH CURVES

VELOCITY - 120 M.P.H.

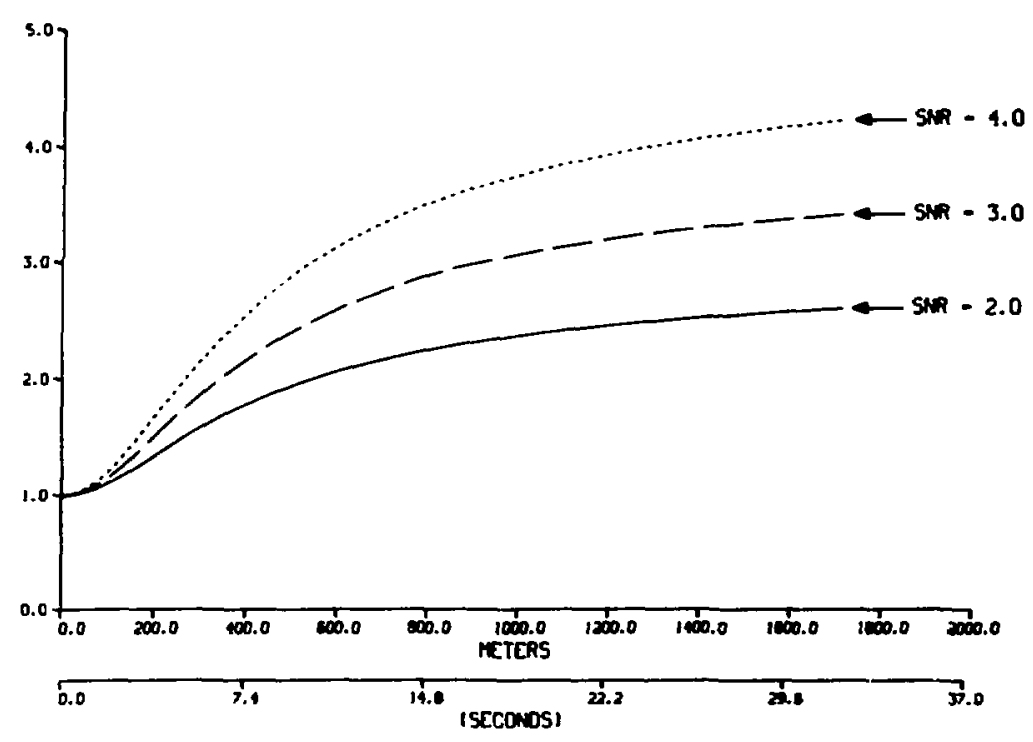

Fig. 13. Increase in the signal-to-noise ratio with segment length. 
E. Relating the Components of Variance in Ground-Based and Aerial Measurements

An important problem in the design of a ground-based sampling experiment is the determination of an adequate sample size. Sample size determination requires information about the variance inherent in ground-based measurements. The suitability of aerial radiometric data as a source of this information will now be considered.

The ground-based measurements ran he thought of as arising from a classical two-stage nested analysis of variance (ANOVA) design:

(1) several "samples" near one "position" and

(2) several "positions" along each "line."

Within this framework, the "samples" are assumed to vary independently with variance $\sigma_{S}^{2}$, and this effect is additive to the "position" effect with variance $\sigma_{P}^{2}$ and the "line" effect with variance $\sigma_{L}^{2}$.

Let $Q_{k}(x, y)$ denote the true concentration, in ppm, at a point $(x, y)$ near the $k$ th flight line. ( $x$ denotes the coordinate along the line, and $y$ is small.) In the ANOVA model, $Q$ is the sum of several components,

$$
Q_{k}\left(x_{i j}, y_{i j}\right)=\mu+L_{k}+P_{k i}+S_{k i j},
$$

where $\left(x_{i j}, y_{i j}\right)$ is the location of the jth sample taken at the $i$ th position, $\mu$ is the overall mean, taken to be constant, and $L_{k}, P_{k i}$ and $S_{k i j}$ are the random effects associated with the line, position and sample, respectively. The random effects model assumes these are all independent, and

$$
\begin{aligned}
& L_{k} \sim n\left(0, \sigma_{L}^{2}\right) \\
& P_{k i} \sim n\left(0, \sigma_{P}^{2}\right) \\
& S_{k i j} \sim n\left(0, \sigma_{S}^{2}\right),
\end{aligned}
$$

where $\eta\left(\mu, \sigma^{2}\right)$ denotes a normal probability distribution with mean $\mu$ and variance $\sigma^{2}$. 
Let $x_{k i j}$ be the measured value of the sample taken at $\left(x_{i j}, y_{i j}\right)$,

$$
x_{k i j}=Q_{k}\left(x_{i j}, y_{i j}\right)+\varepsilon_{k i j},
$$

where the measurement error $\varepsilon_{k i j}$ has a $\eta\left(0, \sigma_{m}^{2}\right)$ distribution.

For a balanced design with $s$ samples per position and $p$ positions per line, the ANOVA tahle is given in Table VI. The $F$ statistic for testing for a line effect will have expected value

$$
I+\frac{s p \sigma_{L}^{2}}{\sigma_{S}^{2}+\sigma_{m}^{2}+s \sigma_{p}^{2}} .
$$

This expected value is bigger than $F$, say, if $\sigma_{L}^{2}>0$ and $s$ and $p$ are so large that

$$
\frac{1}{p} \frac{\sigma_{p}^{2}}{\sigma_{L}^{2}}+\frac{1}{s p} \frac{\sigma_{S}^{2}+\sigma_{m}^{2}}{\sigma_{L}^{2}}<\frac{1}{F-1} .
$$

TABLE VI

ANALYSIS OF VARIANCE FOR GROUND SAMPLES

Sum of Squares

$$
\begin{aligned}
& \sum_{k, i} \sum_{j}\left(x_{k i j}-\bar{x}_{k i .}\right)^{2} \\
& \sum_{k i}\left(\bar{x}_{k i .}-\bar{x}_{k \ldots}\right)^{2} \\
& \sum_{k}\left(\bar{x}_{k \ldots}-\bar{x}\right)^{2}
\end{aligned}
$$

Degrees of Freedom

$\operatorname{lp}(s-1)$

$\ell(p-1)$

\& -1

\section{Expected Mean Square}

$$
\sigma_{S}^{2}+\sigma_{m}^{2}
$$

$$
\sigma_{\mathrm{S}}^{2}+\sigma_{\mathrm{m}}^{2}+s \sigma_{\mathrm{P}}^{2}
$$

$\sigma_{S}^{2}+\sigma_{m}^{2}+s \sigma_{p}^{2}+s p \sigma_{L}^{2}$ 
The problem considered below is the possibility of learning something about the relative magnitures of $\sigma_{S}^{2}, \sigma_{m}, \sigma_{\mathrm{P}}^{2}$ and $\sigma_{\mathrm{L}}^{2}$ from the aerial data. If the ratios on the left-hano side of Eq. (27) could be estimated, then the sample size required to demonstrate a "line effect" could be determined. Unfortunately, it turns out that only the ratio $\sigma_{\mathrm{P}}^{2} / \sigma_{\mathrm{L}}^{2}$ is available from the aerial data. Furthermore, preliminary work on uranium concentrations in the water and sediments of several Rocky Mountain quadrangles suggests that $\sigma_{S}^{2}$ is far from negligible (though possibly $\sigma_{\mathrm{m}}^{2}$ is very small). Therefore, if $s$ and $p$ are chosen under the assumption that the second ratio is negligible, the result will be much too small.

To make the connection between ground-based and aerial measurements, we will now consider $Q_{k}(x, y)$ as a random variable defined along a flight line, and not only at the sampling positions. As in Sec. III.A., we will assume that $Q_{k}(x, y)$ is a second-order stationary process. (Stationarity is, perhaps, improbable, but the development which follows, based on these assumptions, gives results which are qualitatively similar to those obtained with more general assumptions, such as the existence of stationary differences only. Moreover, in the experimental situation being considered here, stationarity within "lines" may be quite a reasonable assumption.)

Parallel to the ANOVA analysis, we write

$$
Q_{k}(x, y)=\mu+L_{k}+P_{k}(x, y)+S_{k}(x, y),
$$

where

$\mu$ is constant over the whole region;

$L_{k}$ is constant along a line, and the values for each line are drawn from a $n\left(0, \sigma_{L}^{2}\right)$ population;

$P_{k}(x, y)$ is a relatively slowly varying quantity, i.e., $\operatorname{Cov}\left[P_{k}(x)\right.$, $\left.P_{k}(x+\Delta x)\right]$ is close to $\sigma_{P}^{2}$ for small $\Delta x$, and does not fall off to zero until $\Delta x$ is several kilcileters, or something on the order of the distance between "positions;"

$S_{k}(x, y)$ is a rapidly varying quantity, $\operatorname{var} S_{k}(x, y)=\sigma_{S}^{2}$, but $\operatorname{Cov}\left[S_{k}(x, y), S_{k}(x+\Delta x, y+\Delta y)\right]$ becomes zero for $\sqrt{\Delta x^{2}+\Delta y^{2}}$ on the order of a few meters. 
As usual, define

$$
C_{Q}(\Delta x, \Delta y)=\operatorname{Cov}\left[Q_{k}(x, y), Q_{k}(x+\Delta x, y+\Delta y)\right]
$$

As a function of $\Delta x$ ( $\Delta y$ held constant), $C_{Q}$ may be quite smooth at the origin, as suggested by Figure 14a, or rather peaked, as in figure 14b. The first case corresponds to little local sampling variation, that is, to $\sigma_{S}^{2}$ being very small. In this case if the "positions" are at least $b$ kilometers apart, $\sigma_{P}^{2}=C_{Q}(0,0)$ is the main component of variance of observations within a line.

Preliminary studies of hydrogeochemical data in the Albuquerque and Montrose quadrangles suggests that figure $14 \mathrm{~b}$ is more typical, however. Figures $15 a$ and $15 b$ are estimates of $\gamma_{Q}(|h|)$ for $\log$ uranium concentrations in sediments and in water in the Montruse quadrangle. [Recall that in the stationary case $\gamma(h)$ is related to the autocovariance function by Eq. (19).] It is clear that $\lim _{h \mid \rightarrow 0} \gamma_{U}(|h|)$ is not zero, and that considerable variability, at least a quarter of the total over the whole quadrangle (the quantity designated as "variance" in the subtitle) can be expected among samples separated by less than 200 meters. Thus, $C_{Q}$ has to be modeled with a substantial discontinuous part, corresponding to $\sigma_{S^{2}}$.

From the aerial data for each flight line, or perhaps an average over all flight lines, an estimate of the autocovariance function $c_{Z}(h)$ can be formed, which is related to $C_{Q}(\Delta x, \Delta y)$ as in Eq. (10):

$$
c_{Z}(h)=\left\{\begin{array}{l}
\varepsilon^{2} \theta^{2}\left[\left(C_{Q}^{*} C_{p}\right)(v h, 0)+C_{B}(h)\right] \quad h \neq 0 \\
\varepsilon^{2} \theta^{2}\left[\left(C_{Q}{ }^{*} C_{p}\right)(v h, 0)+C_{B}(h)\right]+E \operatorname{var} n(t), h=0 .
\end{array}\right.
$$

Here we have assumed that the emissions $\Lambda(x, y)$ are related to the concentration $Q(x, y)$ uy a proportionality factor $\theta$,

$$
\Lambda(x, y)=\theta Q(x, v)
$$


Assume that the background contribution is negligible. From Eq. (29), the contributions to $Q$ which are not constant within a flight line (the only terms which contribute to $C_{Q}$ ) come from $P_{k}$ and $S_{k}$, so

$$
C_{z}(h) \sim \varepsilon^{2} \theta^{2}\left[\left(C_{p}{ }^{*} C_{p}\right)(v h, 0)+\left(C_{S}{ }^{*} C_{p}\right)(v h, 0)\right], \quad h \neq 0 .
$$

The effect of convolution with $c_{p}$ is to broaden the original convolution function $\left(C_{p}\right.$ or $C_{S}$ ) by the width of $C_{p_{2}}$ (about $500 \mathrm{~m}$ ) and to multiply it by the volume under $C_{p}$, which is $P_{v}^{2}$, in the notation of Eq. (5). Assuming $C_{P}$ represents the continuous part of the autocovariance function $C_{Q}$, and ${ }_{\Delta x}, \lim _{\Delta y \rightarrow 0} C_{p}(\Delta x, \Delta y)=\sigma_{p}^{2}$, the first summand in Eq. (29) is approximately

$$
\varepsilon^{2} \theta^{2}\left(C_{P}^{*} C_{p}\right)(v h, 0) \sim \varepsilon^{2} \theta^{2} P_{v} \sigma_{P}^{2}
$$

for small $\mathrm{h}$. On the other hand, $\mathrm{C}_{\mathrm{S}}$ is the discontinuous part of $\mathrm{C}_{\mathrm{Q}}$, and convolution with $C_{p}$ results in a term

$$
\varepsilon^{2} \theta^{2} \sigma_{S}^{2} C_{p}(v h, 0)
$$

for small h. (See remarks in Sec.III.A in connection with Fig. 6b.) Now, $c_{p}(0,0) \ll P_{v}$, so unless $\sigma_{S}^{2}$ is extremely large, the contribution of the second term in Eq. (29) is negligible, and

$$
\hat{c}_{Z}(\Delta h) \sim \varepsilon^{2} \theta^{2} P_{v} \sigma_{P}^{2}
$$

(Compare Eq. (23); again we are using $c_{Z}(\Delta h)$ as an estimate of $h+i p$ $c_{Z}(h)$.) The overall variance of the aerial data over all flight lines includes an additional term, which is $\varepsilon^{2} \theta^{2} P_{v} \sigma_{L}^{2}$,

$$
\operatorname{Var} z \sim \varepsilon^{2} \theta^{2} P_{v}^{2}\left(\sigma_{L}^{2}+\sigma_{P}^{2}\right)+E(\operatorname{Var} \eta)
$$


The average variance within a line, on the other hand, is

$$
\overline{\operatorname{Var} Z_{k}}-\varepsilon^{2} \theta^{2} P_{v}^{2} \sigma_{P}^{2}+E(\operatorname{Var} \eta)
$$

Combining Eqs. (30), (31) and (32) we can arrive at an estimate of the ratio $\sigma_{\mathrm{P}} / \sigma_{L}^{2}$. However, as we saw above, a term involving $\sigma_{S}^{2}$ is seldom available. Some experiments to determine $\sigma_{S}^{2}$ directly are therefore necessary. 


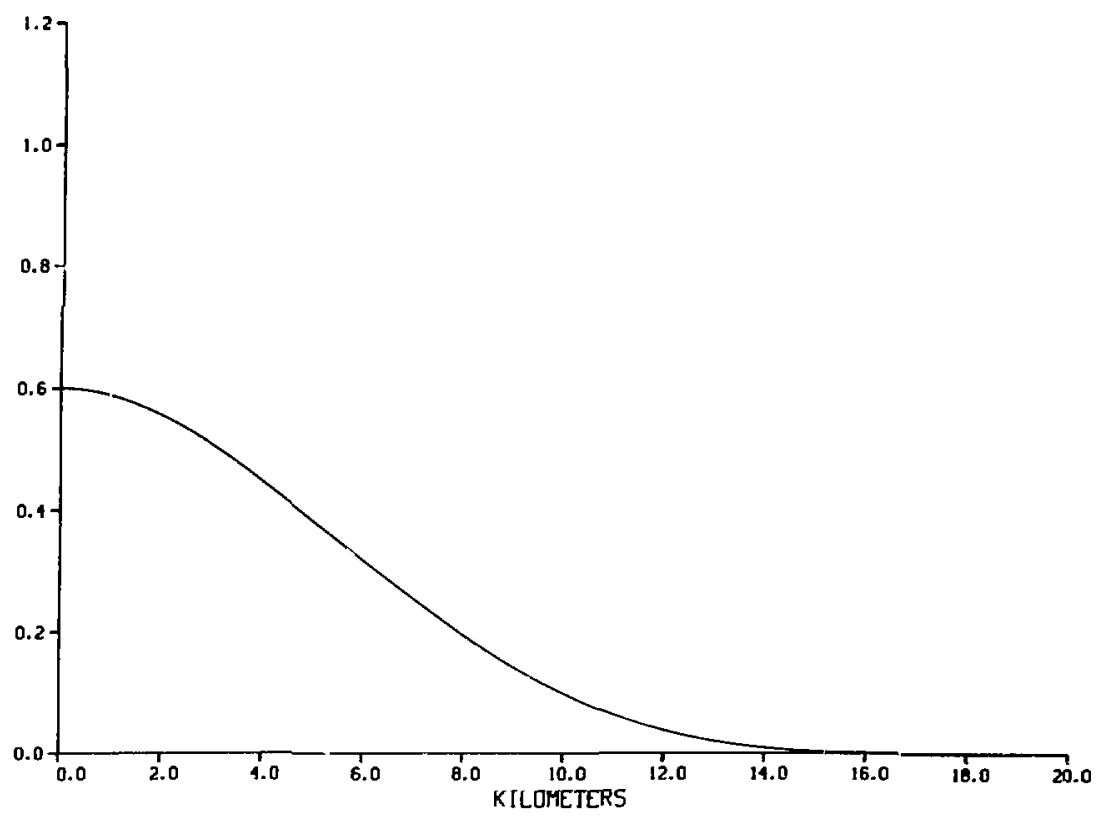

Fig. 14a. Possible shape for autocovariance function of a smooth process.

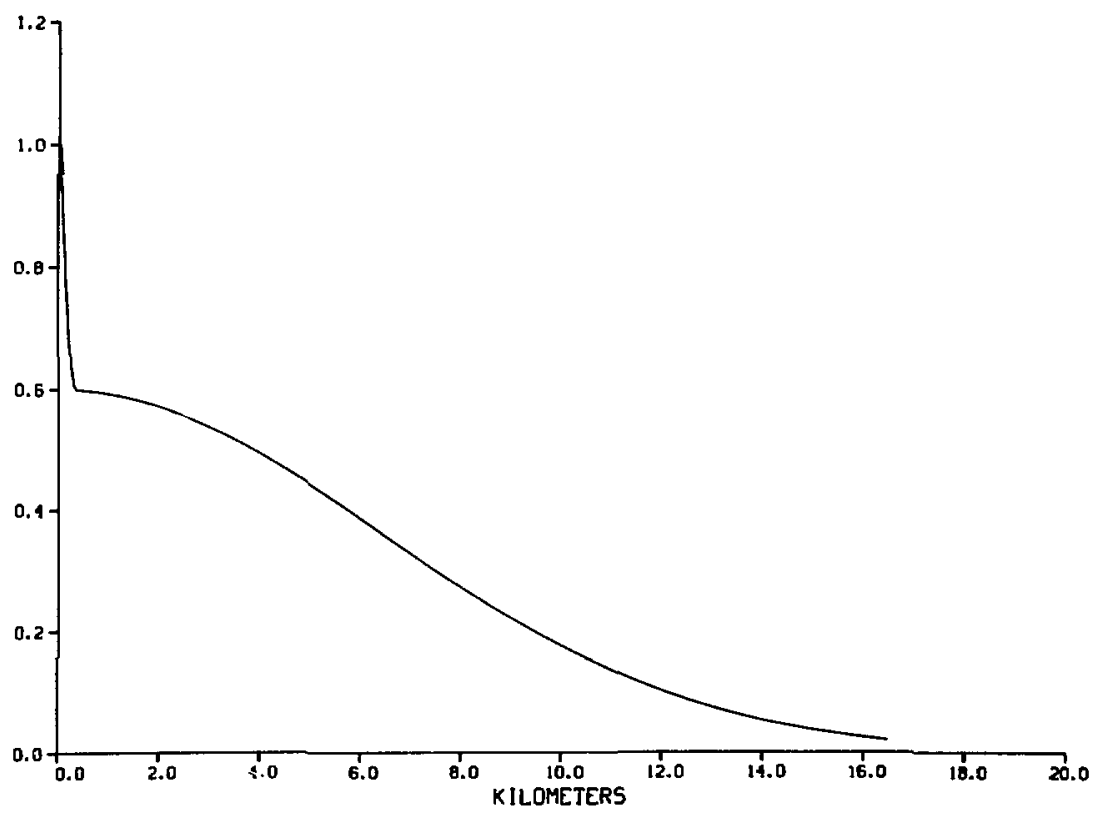

Fig. 14b. Possible shape for autocovariance function of a noisy process. 
URANIUM IN SEDIMENTS, MONTROSE OUADRANGLE (LOG)

VFRIFACC - .458

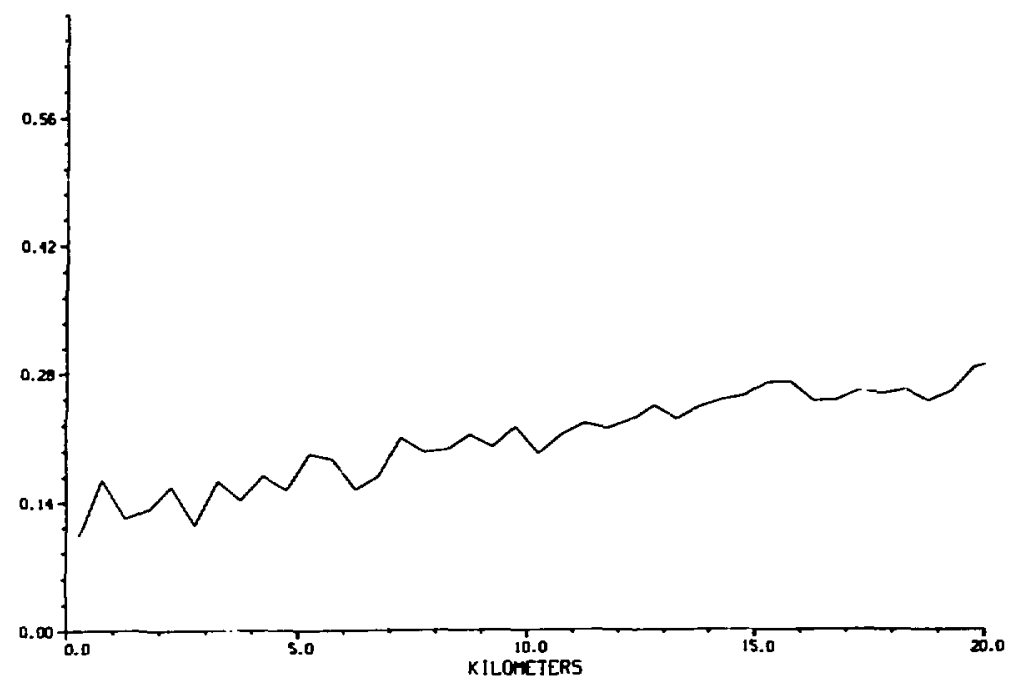

Fig. 15a. Experimental variogram of the logs of uranium concentration in sediments.

URANIUM IN WATER, IAONTROSE OUADRANGLE ILOGI

VRRIANCE - 2.401

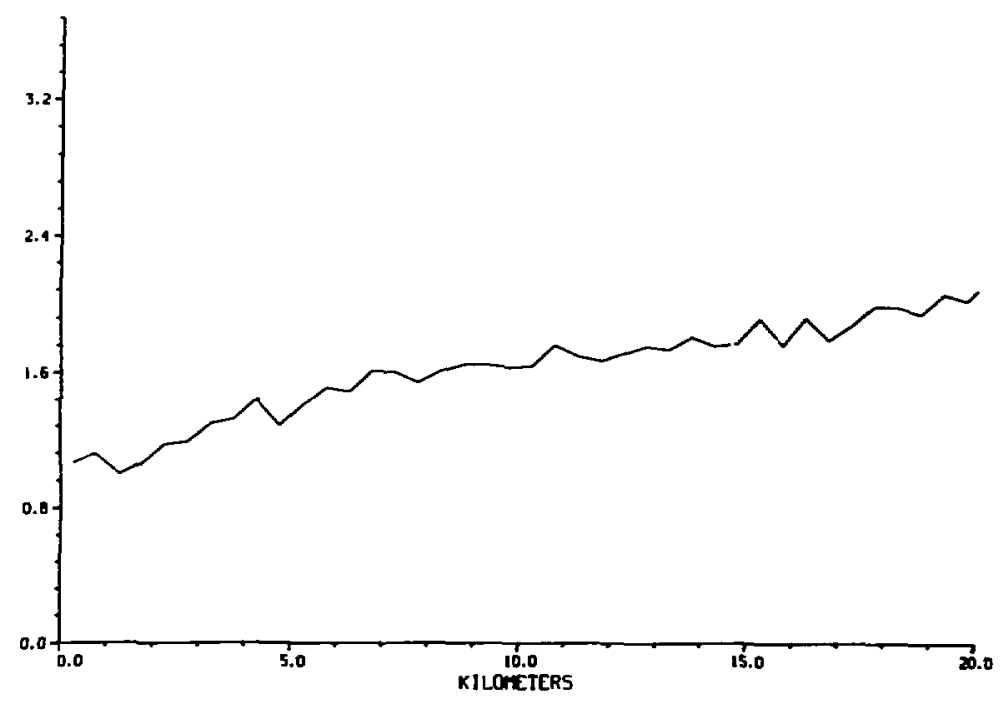

Fig. 15b. Experinental variogram of the logs of uranium concentration in water. 
IV. COMPARISON OF NORMAL AND LOGNORMAL METHODS OF PERCENTILE ESTIMATION

Implicitly or explicitly, percentile estimation has always been an important feature of the treatment of National Uranium Resource Evaluation (NURE) aerial radiometric data. Standard deviation maps actually show the location of records which fall outside certain percentile ranges if one assumes the data follow either a normal or lognormal distribution. The definition and identification of anomalous points in regions is usually very closely related to the estimation of percentiles.

Because of the prominance of percentiles in the analysis of NURE aerial radiometric data, a study was conducted to compare two commonly used methods of estimating them. The main thrust of the study was to determine how estimation is influenced by specific types of departures from distributional assumptions and to provide a suggested method for estimating percentiles. A report presenting the results of this study will be released by DOE, Grand Junction, in May or June, 1980. Highlights are presented here.

The two methods of estimation consicered are (1) the normal assumption method and (2) the lognormal assumption method. In general, if a random sample is known to have come from a particular type of distribution, the best way to estimate percentiles is to use the properties of that distribution. The two methods assume that the data are from either a normal or lognormal distribution and make use of the properties of these distributions.

Whenever a procedure is applied which, either implicitly or explicitly, is based on a particular probability distribution, the user should be aware of the consequences of failure to meet mathematical assumptions. For instance, under normal distribution theory, $\bar{x}+1.6455$ provides an estimate of the 95th percentile, where $\bar{x}$ and $S$ are the sample mean and standard deviation. (The quantity 1.645 is obtained from a normal table.) The meaning of $\bar{x}+1.6455$ is uncertain, however, if the distribution from which the sample was taken is not normal.

There are many ways in which distributional assumptions can be violated and we consider two specific cases.

(1) assuming that a distribution is lognormal when it is really normal, and 
(2) assuming that a distribution is normal when it is really lognormal.

Figures 16 and 17 summarize the results of a Monte Car'o study to determine the percentage error realized by using the wrong distribution. The error realized depends on two variables: the percentile being estimated and the coefficient of variation ( $\mathrm{CV}$ ) of the population being sampled. (The coefficient of variation is the ratio of the standard deviation to the mean.)

The curves in Fig. 16 show those $C V$-percentile combinations for which there is a $5 \%$ error when the wrong distribution is used. The regions where errors are greater than and less than 5\% are also indicated. Figure 16 shows that making an error by assuming the data come from a normal population when they really come from a lognormal population causes problems primarily when estimating tail percentiles. When estimating upper percentiles, however, the error of making a log-transformation has more serious consequences than does the error of failing to transform. When estimating lower tail percentiles, the error of failing to perform a log-transformation has much more serious consequences than does the error of transforming. When one is estimating percentiles that are between the loth and 80th, the consequence of either type of error is not great if the coefficient of variation is reasonably small, say less than 0.27 .

Figure 17 provides some more detail for the upper percentiles. The 5\% error line resulting from the failure to take logs is shown. The $10 \%$ error line for erroneously taking logs is also shown. For coefficients of variation between 0.32 and 0.27 , the error of assuming normality causes an error of less than $5 \%$ when estimating the 90th and 95th percentiles while the error of assuming lognormality causes at least a $10 \%$ error.

Based on the above observations the following suggestions can be made. These are general rules of thumb and should not be interpreted to be precise directions for percentile estimation. As throughout the development, they assume that normal and loanormal ponulations are the only possibilities.

(1) The coefficient of variation should be computed. If the coefficient is large, say greater than 0.27 , percentile results should be interpreted cautiously. 
(2) If a choice between the two distributions cannot be made and if the luwer percentiles (10th and below) are of interest, the safest course of action $i$ is to assume lognormality. If the central percentiles (10th to 80th) are of interest, it makes little difference which distribution is chosen. When the upper percentiles are of interest, the safest course of action to take when in doubt is to assume the normal distribution. All of these remarks assume that the coefficient of variation is in the favorable range defined above.

(3) Because of the large errors which can result from the choice of the wrong distribution, an effort should be made to determine which distribution best fits a set of data. A chi-square, kolomogorov, Lilliefors, or other test for normality (Ref. 5) should be performed. This is particularly important in the case of a large coefficient of variation. 


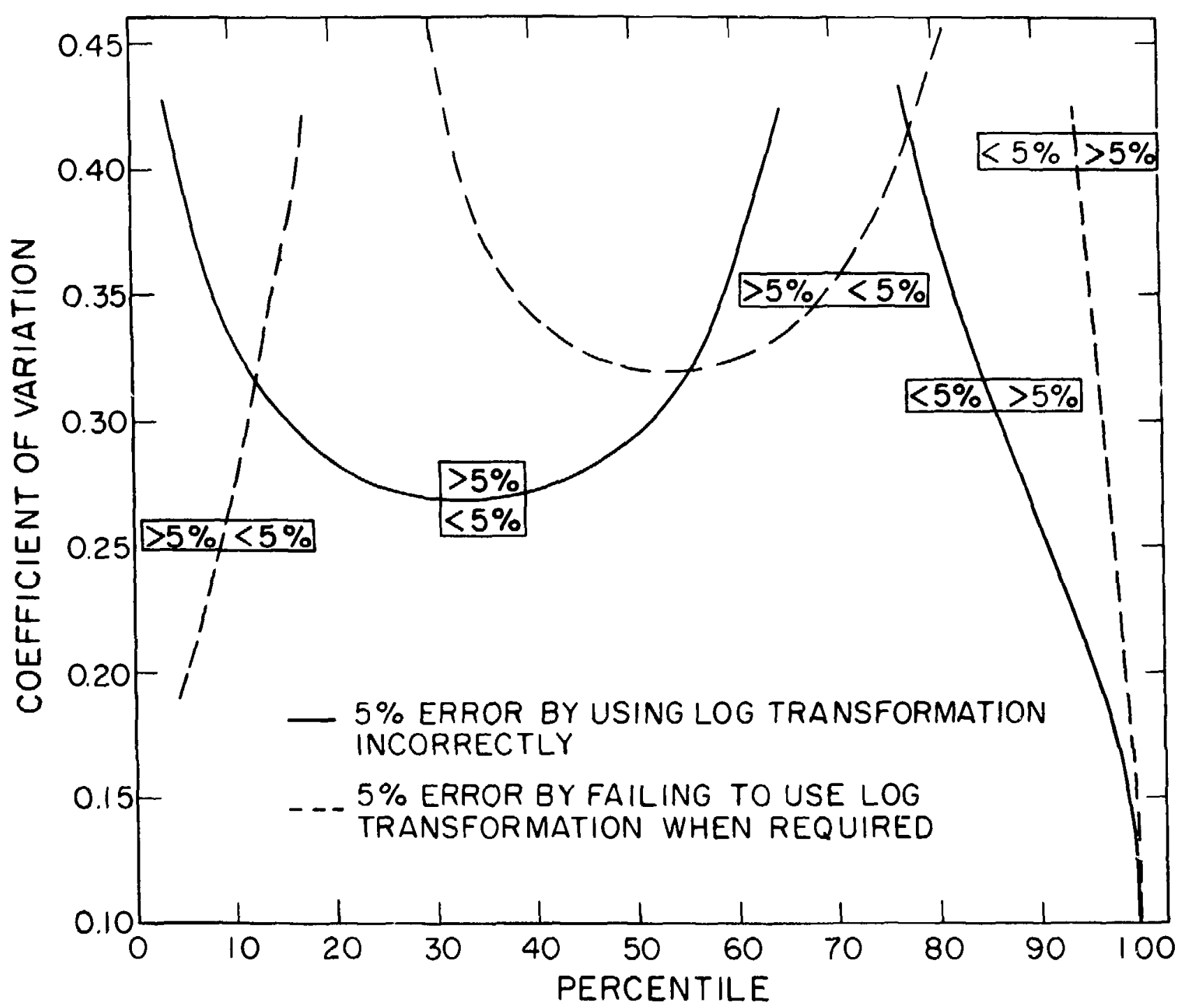

Fig. 16. Coefficient of variation/percentile combinations for which there is a $5 \%$ error when using the wrong distribution. 


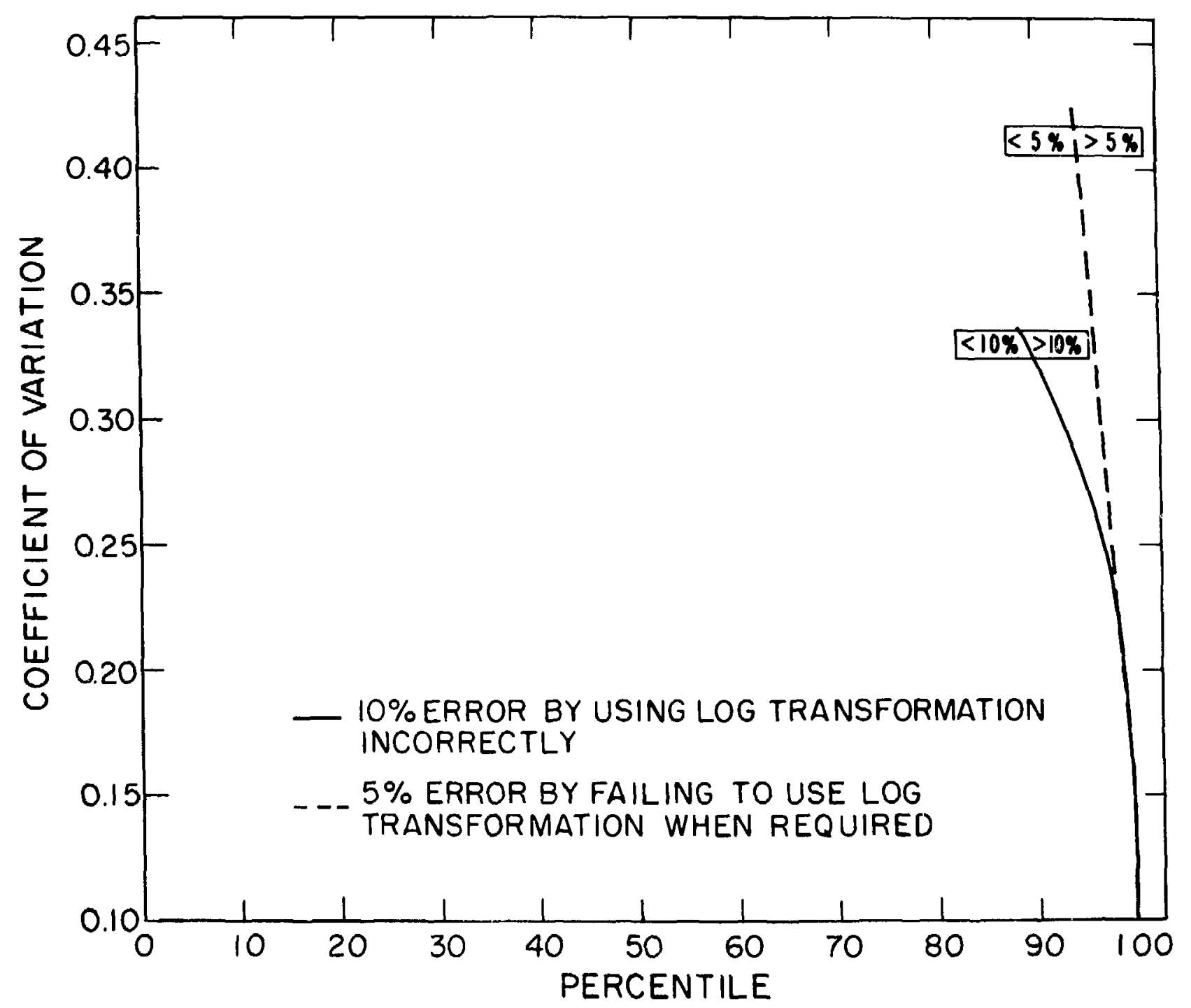
transforming. 
V. URANIUM FAVORABILITY INDEX

During the reporting period computer programs were developed to efficiently and accurately process large sets of data. These codes were applied to the data collected on the 22 quadrangles listed below.

$\begin{array}{ll}\text { Rawlins } & \text { Sherman } \\ \text { Casper } & \text { Pratt } \\ \text { Greeley } & \text { Rice Lake } \\ \text { Mesa } & \text { Ashland } \\ \text { Rock Springs } & \text { Eau Claire } \\ \text { Enid } & \text { Green Bay } \\ \text { Harrisburg } & \text { Hutchinson } \\ \text { Scranton } & \text { Lamar } \\ \text { Tyonek } & \text { Manhattan } \\ \text { Mt. Mckinley } & \text { Talkeetna } \\ \text { Lime Hills } & \text { Wichita }\end{array}$

The data base containing output from these codes plus the original data now consists of approximately 30 million 60-bit words of information.

\section{A. Code Development}

We have developed a program which finds percentiles for data sets of up to 200,000 records. The algorithm, which is based on the Blum algorithm (Ref. 5), can be summarized as shown below. The algorithm returns the value of the Th largest number from a set of size $n$.

(1) Select a sample of 1000 equally spaced records from the original data. Store the sample in an array, $Y$, in ascending order.

(2) From this sample, estinate the location of the Th largest element in the original data. Cutoffs of $5 \%$ above and below this estimate are computed. Compute:

$$
\begin{aligned}
& \text { lower } \text { index }=\max \left(\frac{N-T}{N} \cdot 1000-50,1\right) \\
& \text { upper index }=\min \left(\frac{N-T}{N} \cdot 1000+50,1000\right) \\
& \text { lower cutoff }=Y(\text { lower index }) \\
& \text { upper cutoff }=Y(\text { upper index }) .
\end{aligned}
$$


(3) Extract from the original data all records between the lower and upper cutoffs. This data will contain approximately $10 \%$ of the original data and will be searched for the true $T^{\text {th }}$ largest record.

(4) Apply the Blum algorithm (Ref. 5) to this reduced data set to compute the Th largest element.

A second program which was developed computes the covariance matrix efficiently and accurately for a large set of data. The algorithm is a one-pass algorithm similar to the one-pass algorithm of West (Ref. 6) for computing the variance of a set of data. Our algorithm is summarized here.

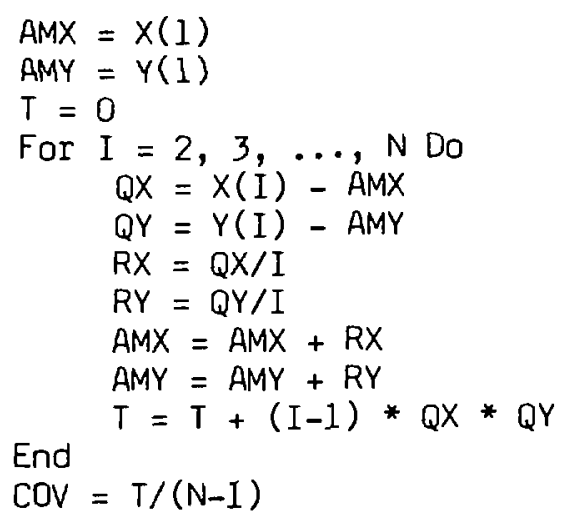

Because of the sizes of the data bases under study, it is not practical to try to read in all of the data and process it in one step as shown above. Instead, we input (from a mass storage device) 10,000 elements at a time for each of the 2 arrays, $X$ and $Y$, and accumulate the necessary information in $T$.

The two codes just described have been sent to BFEC, Grand Junction, Colorado, for their use.

\section{B. Large Data Base}

For each of the 22 quadrangles we have computed 265 statistics based on the following 10 variables: thallium, bismuth, potassium, gross count, $T l / g r o s s, B i / g r o s s, ~ K / g r o s s, ~ B i / K, B i / T l$, and $T l / K$. For each variable we have computed the median, mean, and standard deviation; the 99th, 95th, 90th, lst, 5th, and 10th percentiles; the average and standard deviation of all the 
points above the 99th, 95th, and 90th percentiles; and the same for all points below the lst, 5th, and loth percentiles. In addition, we have computed the 55 upper triangular elements of the 10 by 10 covariance matrix for the variables listed above. Thus, there are 10 variables times 21 statistics computed on them plus the 55 elements of the covariance matrix for a total of 265 statistics.

Work is underway to begin analysis of this data in order to find a uranium favorability index.

\section{Problems with the Data}

Some of the quadrangles listed in Ref. 7 are not being used in this study. Some of the reasons for their omission are missing data, incomplete quadrangle coverage, and difficulties in reading the data tapes.

\section{REFERENCES}

1. T. R. Bement and M. S. Waterman, "Locating Maximum Variance Segments in Sequential Data," Math. Geol. 9, 55-61 (1977).

2. A. G. Journel and C. J. Huijbregts, Mining Geostatistics (Academic Press, London, 1978).

3. J. Tammenmaa, R. L. Grasty and M. Peltoniemi, "The Reduction of Statistical Noise in Airborne Radiometric Data," Canadian Journal of Earth Sciences 13, 1351-1357 (1976).

4. K. L. Kosanke and C. D. Koch, "An Aerial Radiometric Data Modeling Program," IEEE Trans. Nuclear Science NS-25, No. 1, 767 (1978).

5. M. Blum, R. W. Floyd, V. Pratt, R. L. Rivest and R. E. Tarjan, "Time Bounds for Selection," Journal Computer and Syst. Sci. I, 448-461 (1973).

6. D. H. D. West, "Updating and Variance Estimates: An Impro:'ed Method," Comm. ACM 22,9, 532-535 (September 1979).

7. J. A. Howell, T. R. Bement and P. L. Buslee, "Geostatistics Project of the National Uranium Resource Evaluation Program, April-September 1979," Los Alamos Scientific Laboratory report LA-8175-PR (December 1979). 